\title{
Fenestrate Bryozoa of the Moniello Formation (Lower-Middle Devonian, NW Spain)
}

\author{
JUAN LUIS SUÁREZ ANDRÉS \& PATRICK N. WYSE JACKSON
}

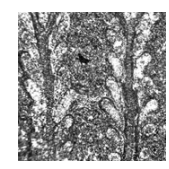

\begin{abstract}
Lower-Middle Devonian non-fenestellid fenestrate bryozoans of NW Spain are reported, from two coastal sections of the upper Emsian-lower Eifelian Moniello Formation, the type locality at Moniello Inlet and at Arnao. Eighteen species of non-Fenestellidae fenestrates belonging to thirteen genera and six families have been identified; nine species are new: Bashkirella arnaoense, Utropora parva, Bigeyina cantabrica, B. spinosa, Fenestrapora elegans, Paraseptopora geometrica, P. irregularis, Kalvariella antiqua and Filites robustus. Fenestrates described herein, together with earlier reported taxa of the Family Fenestellidae from the Moniello Formation show palaeobiogeographic affinities with those of neighbouring Gondwanan regions, especially with Bohemia, but also with Middle Devonian assemblages from Eastern North America. The fenestrate fauna from NW Spain comprises seven species common with the Lower Devonian of the Prague Basin (Bohemia, Czech Republic) and genera with relatively limited geographic distributions, such as Utropora and Filites. A remarkable feature of the studied fauna is the presence of several species that represent the earliest and westernmost reported occurrence of their genera, Bashkirella, Paraseptopora and Kalvariella, as well as previously reported Cavernella asturica. The occurrence of these species together with the stratigraphic and geographic position of previously reported ones indicate the existence of large gaps in the distribution of these Lazarus genera. $\bullet$ Key words: Fenestrates, Emsian, Eifelian, taxonomy, diversity, palaeobiogeography, Lazarus taxa.
\end{abstract}

SUÁREZ ANDRÉS, J.L. \& WYSE JACKSON, P.N. 2017. Fenestrate Bryozoa of the Moniello Formation (Lower-Middle Devonian, NW Spain). Bulletin of Geosciences 92(2), 153-183 (9 figures, 16 tables). Czech Geological Survey, Prague. ISSN 1214-1119. Manuscript received March 9, 2017; accepted in revised form April 25, 2017; published online June 23, 2017; issued June 30, 2017.

Juan Luis Suárez Andrés, SONINGEO, S.L. Parque Científico y Tecnológico de Cantabria, C/Isabel Torres, 9 - 39011, Santander,Spain; juanl_suarez@yahoo.es・Patrick N. Wyse Jackson, Department of Geology, Trinity College, Dublin 2,Ireland;wysjcknp@tcd.ie

Bryozoans are common among benthic faunas throughout most of the Palaeozoic. Their associations tend to be dominated by members of the orders Trepostomata and Cystoporata during the Early Palaeozoic, while those of the orders Fenestrata and Cryptostomata are usually predominant in the Late Palaeozoic (Ernst 2013). The studies devoted to the Devonian bryozoan fauna of NW Spain during the last number of years reveal a well-represented, highly diverse group providing important palaeobiogeographic and palaeobiologic data (Ernst 2010, 2011, 2012; Suárez Andrés \& McKinney 2010; Ernst et al. 2011; Ernst \& Buttler 2012; Suárez Andrés \& Wyse Jackson 2014; Suárez Andrés et al. 2014; Suárez Andrés \& Ernst 2015). The Lower-Middle Devonian succession of NW Spain provides a rich record of episodes of reefal development, an outstanding evidence of high biodiversity that has received close attention for decades (Méndez-Bedia 1976, Méndez-Bedia et al. 1994, Soto et al. 1994, Fernández et al. 2006, Fernández-Martínez et al. 2006, among others). Bryozoans are locally very abundant and fenestrates gene- rally constitute the most conspicuous group, but their composition has only been studied in detail in recent studies.

This paper is a contribution to the knowledge of the Devonian fenestrate fauna from NW Spain with a focus on the systematic composition and the palaeobiogeographic relationships of the representatives of the order Fenestrata from the upper Emsian-lower Eifelian Moniello Formation. Eighteen species and thirteen genera belonging to six families are reported herein from the Moniello Formation (Asturias, northern slope of the Cantabrian Mountains); nine of these species are new. These data, along with those reported in previous works, are used to assess the palaeobiogeographic affinities of the Lower-Middle Devonian Spanish fenestrates.

\section{Geological setting}

The bryozoan fauna described herein belongs to the Moniello Formation, a calcareous unit within the Cantabrian 
Zone of the Iberian Massif (Fig. 1), which represents the external zone of the Variscan Orogen (Alonso et al. 2009). The Cantabrian Zone constitutes a part of the provinces of Asturias and León, (NW Spain), with a thick Palaeozoic succession affected by thin-skinned tectonics (PérezEstaún et al. 1988). The Moniello Formation is a 200-300 m thick unit composed of limestones and marls, outcropping at the northern slope of the Cantabrian Mountains. The age of this unit is late Emsian-early Eifelian (Lower-Middle Devonian), between the Polygnathus serotinus and the $P$. costatus partitus zones (García-López \& Sanz-López 2002, García-López et al. 2002). Its lateral equivalent at the southern slope is the Santa Lucía Formation (Comte 1936). Méndez-Bedia (1976) described the bio- and lithofacies present in the Moniello Formation and reported transition from coastal to platform and reefal environments from the eastern sections of the unit towards the west. Arbizu et al. (1979) formally described the Moniello Formation and its biostratigraphy. The reefal facies have been studied by the Research Group on Devonian Reefs of the University of Oviedo (Méndez-Bedia et al. 1994, Soto et al. 1994, Fernández et al. 2006, Fernández-Martínez et al. 2006).

Two outcrops located on the central coast of Asturias and corresponding to the outermost carbonate platform, reefal and peri-reefal facies (Méndez-Bedia 1976) have been sampled for this study. The first is the type section, exposed immediately westward of the Moniello Inlet. The second one is a discontinuous outcrop between the localities of Salinas and Arnao, which is the westernmost and thus the most distal section of the Moniello Formation along the coast of Asturias (Fig. 1). The Arnao section shows incomplete outcrops of this unit strongly affected by tectonics, covered by overlying disconformable deposits. This section has been documented for decades due to the development of reefal facies including a well-preserved bioherm (Méndez-Bedia 1976; Sánchez de la Torre \& Manjón 1976; Méndez-Bedia \& Soto 1984; Soto \& Méndez-Bedia 1985; Arbizu et al. 1991; Arbizu \& Méndez-Bedia 2006a, b).

\section{Material and methods}

The Moniello and Arnao outcrops of the Moniello Formation were sampled for fenestrate bryozoans. Most of the specimens are preserved in calcite, though partial silicification is relatively common. Specimens were sectioned and acetate peels were prepared of three standard oriented sections, whenever possible. At least two tangential sections at different depths were prepared to observe morphological details; this procedure is generally advisable for fenestrates, but especially relevant for appreciation of characteristics of the superstructures developed above the obverse surfaces of zoaria in the Semicosciniidae. Samples were observed under optical microscope and digital imaging was carried out; morphometric characters were measured on digital images by means of the software Image J.

In contrast with other fenestrate families, the Semicosciniidae frequently show very distinct variations in chamber size and morphology depending on the position of autozooecia relative to fenestrules and dissepiments. These morphological variations are documented in the description of species, and separate morphometric measurements are given for chambers developed adjacent to fenestrules and to dissepiments and for those not affected by these interactions. Characters, such as the maximal chamber width or chamber length are specifically documented (see the corresponding tables). In species with sinuous branches (i.e. Bigeyina victrola and Fenestrapora elegans sp. nov.), measurements of branch spacing have been treated in two separate groups, those adjacent to fenestrules and adjacent to dissepiments, to reflect bimodality. In pinnate species (families Septoporidae and Acanthocladiidae), zoarial and zooecial characters were measured on main branches and pinnae and tabulated separately. One hundred forty specimens were studied for the systematic treatment presented herein. One hundred thirty of them were adequately described and figured, while the rest (all three specimens of Prolixicella sp. and ten specimens assigned to Anastomopora sp.) have been identified to the genus level only, due to their poor state of preservation and/or the small number of specimens obtained, and have been briefly described but not figured. Most species have been identified in both outcrops, except for Eosemicoscinium aff. burultobense, found only in Moniello Inlet, and Paraseptopora geometrica, Penniretepora bohemica and Bashkirella arnaoense, in Arnao.

All specimens are deposited at the Department of Geology of the University of Oviedo. Accession numbers (DGO) for the species described in this paper are indicated within the systematic description.

\section{Systematic Palaeontology}

Phylum Bryozoa Ehrenberg, 1831

Class Stenolaemata Borg, 1926

Order Fenestrata Elias \& Condra, 1957

Suborder Phylloporinina Lavrentjeva, 1979

Family Phylloporinidae Ulrich, 1890

\section{Genus Prolixicella Ernst \& Schroeder, 2007}

Type species. - Prolixicella bifurcata Ernst \& Schroeder, 2007; Givetian, Germany. 


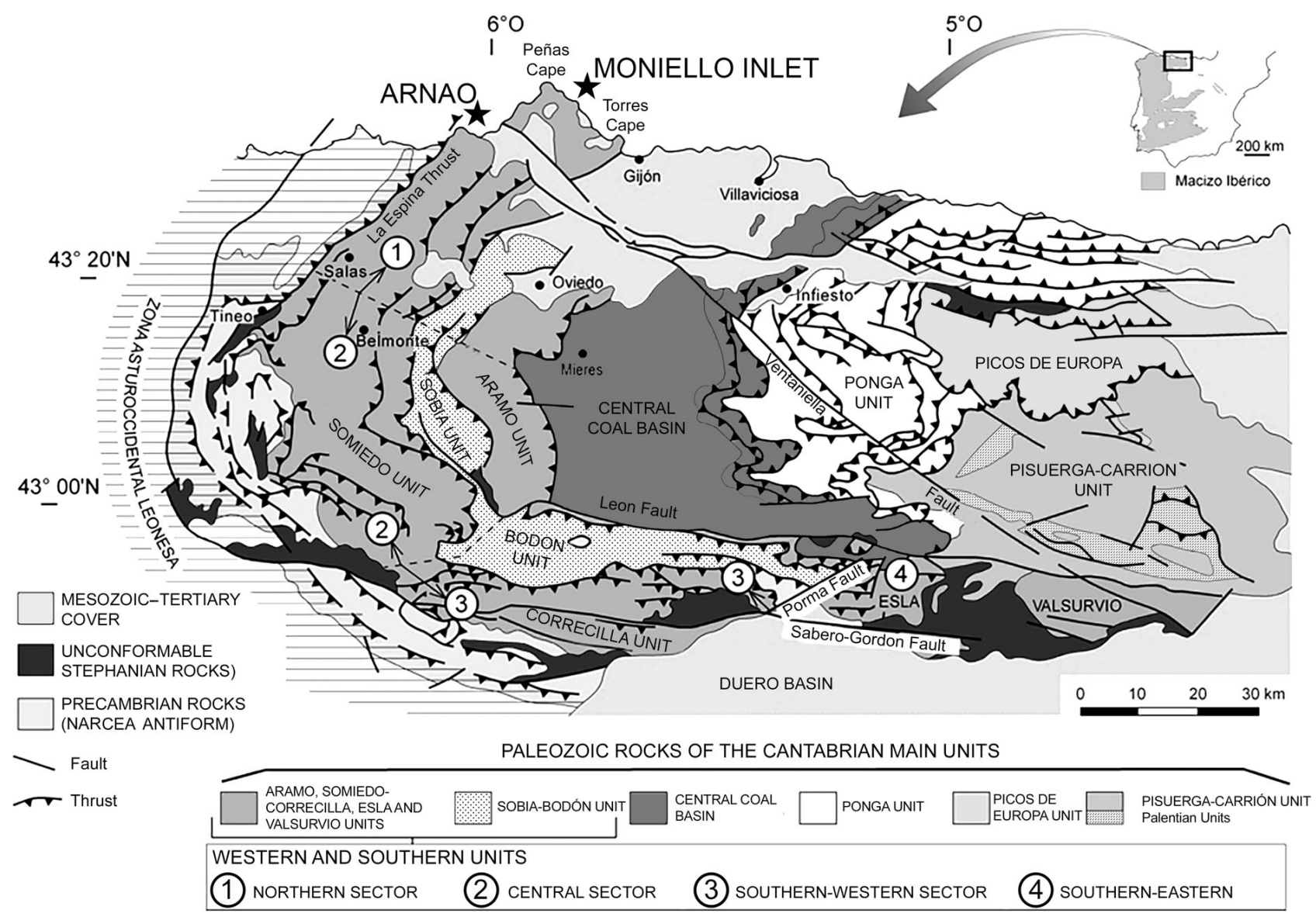

Figure 1. Geological map of the Cantabrian Zone after Pérez Estaún et al. (2004) showing the location of the Arnao and Moniello outcrops, Asturias, NW Spain.

\section{Prolixicella sp.}

Material. - Three small specimens, DGO 13032-13034; one sectioned, recrystallized.

Description. - Exterior: Zoarium dendroid; multiserial branches straight to gently curved, bifurcating, anastomoses absent. Branch width $0.30-0.64 \mathrm{~mm}$, average $0.46 \mathrm{~mm}$.

Interior: Autozooecial chambers tubular, very long. Hemisepta and heterozooecia not observed. Macrostyles present on the reverse surface of branches.

Remarks. - Only one of the three small specimens collected has been sectioned, and internal features cannot be clearly appreciated due to recrystallization. Good quality images illustrating zooecial characters could not be obtained, so the species is not figured herein. The number, size and preservation of specimens allow for assignment to Prolixicella but prevent unequivocal species identification of the Spanish material; thus this material is kept in open nomenclature. Ernst \& Schroeder (2007) provided the diagnosis of Prolixicella, reporting the absence of heterozooecia according to observations on its type species, $P$. bifurcata, from the Givetian of Germany. Ernst (2012) described two new species, P. ibera and P. parva, from the Emsian of NW Spain and emended the original diagnosis to reflect the presence of apparent reproductive heterozooecia in $P$. parva. Prolixicella sp. has narrower branches than P. ibera and P. parva. The species defined by Ernst (2012) represent the earliest record of this genus and the only one from the Early Devonian.

Occurrence. - Lower Eifelian. Arnao, Cantabrian Zone, NW Spain.

Family Chasmatoporidae Shulga-Nesterenko, 1955

\section{Genus Bashkirella Nikiforova, 1939}

Type species. - Bashkirella ornata Nikiforova, 1939; Lower Permian, Urals, Russia.

Bashkirella arnaoense sp. nov.

Figure 2A-E, Table 1

Holotype. - DGO 13025. 
Table 1. Measurements of Bashkirella arnaoense sp. nov. X: mean; SD: standard deviation; $\mathrm{CV}$ : coefficient of variation; Max: maximal value; Min: minimal value; $\mathrm{N}$ : number of measurements.

\begin{tabular}{|c|c|c|c|c|c|c|}
\hline & $X$ & SD & $\mathrm{CV}$ & MAX & MIN & $\mathrm{N}$ \\
\hline Branch width (mm) & 0.75 & 0.155 & 0.0240 & 1.02 & 0.42 & 23 \\
\hline Fenestrule length $(\mathrm{mm})$ & 2.83 & 0.265 & 0.0702 & 3.07 & 2.34 & 8 \\
\hline Fenestrule width (mm) & 1.77 & 0.312 & 0.0976 & 2.38 & 1.57 & 6 \\
\hline $\begin{array}{l}\text { Aperture spacing across } \\
\text { branch }(\mathrm{mm})\end{array}$ & 0.43 & 0.014 & 0.0002 & 0.42 & 0.44 & 2 \\
\hline Aperture width (mm) & 0.12 & 0.007 & 0.0001 & 0.13 & 0.11 & 4 \\
\hline Aperture length (mm) & 0.21 & 0.005 & 0.0001 & 0.21 & 0.20 & 4 \\
\hline $\begin{array}{l}\text { Maximal chamber width } \\
(\mathrm{mm})\end{array}$ & 0.19 & 0.034 & 0.0011 & 0.25 & 0.13 & 17 \\
\hline
\end{tabular}

Paratypes. - DGO 13026, 13029.

Type horizon and locality. - Lower Eifelian, Moniello Formation. Arnao.

Material. - Seven specimens, DGO 13025-13031; four sectioned.

Etymology. - From the Asturian village of Arnao, the type locality. Gender neuter.

Diagnosis. - Bashkirella with wide branches and largely spaced, oval apertures. Fenestrules large, highly variable in size.

Description. - Exterior: Zoarium irregularly reticulate, branches anastomosed. Fenestrules medium to large, irregular in shape and size. Apertures oval, arranged in 3-5 rows.

Interior: Autozooecial chambers elongate, sack-shaped to tubular, arranged in 3-5 rows around a weakly defined axis. Moderately short vestibule. Hemisepta absent; terminal diaphragms may occur. Prominent styles between zooecial apertures and on the reverse of branches. Laminar skeleton moderately thickened on the reverse of branches. Modified irregular zooecia, with larger and shorter chambers than those of regular autozooecia, may occur at anastomoses.

Remarks. - Bashkirella arnaoense is the earliest reported species of this genus. Two other Devonian species had been previously described, B. devonica (Dessilly, 1967) from the upper Eifelian of Belgium and Germany (Ernst \& Schroeder 2007) and B. bifurcata (Gorjunova, 1966) from the upper Eifelian of Armenia. Both species have thinner branches and closer autozooecial aperture spacing than B. arnaoense. Fenestrules of B. arnaoense are larger than those of the other Eifelian species. Fenestrules in Bashkirella are too irregular in shape and size to consider mean values statistically significant for species description and comparison; minimal and maximal values might be used instead to provide some sense of morphological variation, but these should not be considered as a critic character for species discrimination.

Occurrence. - Lower Eifelian. Arnao, Cantabrian Zone, NW Spain.

Suborder Fenestrina Astrova \& Morozova, 1956

Family Semicosciniidae Morozova, 1987

\section{Genus Utropora Počta, 1894}

Type species. - Fenestella (Utropora) nobilis Barrande, 1894 in Počta (1894); Lower Devonian, Prague Basin (Czech Republic).

Utropora parva sp. nov.

Figure 2F-J, Table 2

Holotype. - DGO 12961.

Paratypes. - DGO 12962-12965.

Type horizon and locality. - Upper Emsian, Moniello Formation. Moniello Inlet.

Material. - Six specimens, DGO 12961-12966.

Etymology. - From Latin parva, small, referring to chamber length. Gender feminine.

Diagnosis. - Utropora with wide branches, relatively small fenestrules and short and wide zooecial chambers.

Description. - Exterior: Zoarium reticulate, fan-shaped. Branches slightly sinuous, dissepiments wide. Keel wide

Figure 2. A-E - Bashkirella arnaoense sp. nov. Moniello Formation. • A - tangential section; enlarged zooecia can be observed at anastomoses, DGO 13026. • B, C - tangential sections, DGO 13025, holotype; B - arrangement of autozooecia and terminal diaphragms sealing apertures (left); C - zooecial apertures and styles. $\bullet$ D - fragmentary specimen showing anastomosed meshwork, DGO 13031 . E - transverse section of two branches, DGO 13029. - F-J - Utropora parva sp. nov. Moniello Formation. - F - transverse section of a branch cutting distal tubes, (arrows). Holotype, DGO 12961. - G - oblique tangential section, DGO 12962. $\mathrm{H}$ - shallow tangential section; the position of the distal tube can be observed. Holotype, DGO 12961. - I - tangential section through the reverse of branches, DGO 12964. • J - longitudinal section, DGO 12964. 
Juan Luis Suárez Andrés \& Patrick N. Wyse Jackson • Lower-Middle Devonian fenestrate Bryozoa (NW Spain)
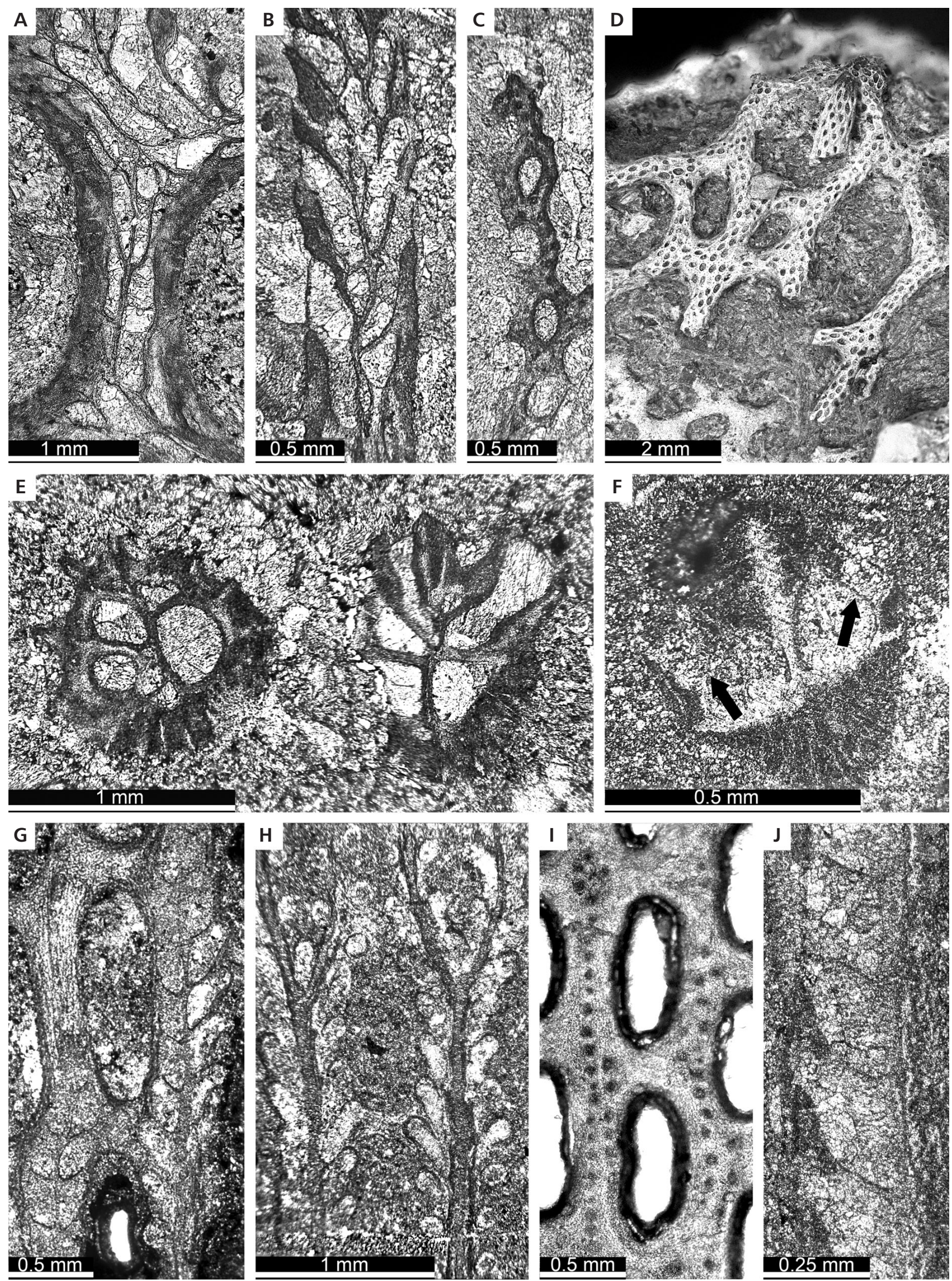
Table 2. Measurements of Utropora parva sp. nov. Abbreviations as in Table 1.

\begin{tabular}{|c|c|c|c|c|c|c|}
\hline & $X$ & SD & $\mathrm{CV}$ & MAX & MIN & $\mathrm{N}$ \\
\hline Branch thickness (mm) & 0.51 & 0.077 & 0.0060 & 0.68 & 0.44 & 17 \\
\hline Branch width (mm) & 0.39 & 0.043 & 0.0019 & 0.49 & 0.32 & 22 \\
\hline Fenestrule length (mm) & 0.90 & 0.133 & 0.0176 & 1.25 & 0.71 & 25 \\
\hline Fenestrule width (mm) & 0.35 & 0.069 & 0.0048 & 0.46 & 0.20 & 23 \\
\hline $\begin{array}{l}\text { Dissepiment width } \\
(\mathrm{mm})\end{array}$ & 0.33 & 0.053 & 0.0028 & 0.45 & 0.25 & 37 \\
\hline $\begin{array}{l}\text { Distance between } \\
\text { branch centres }(\mathrm{mm})\end{array}$ & 0.67 & 0.095 & 0.0090 & 0.88 & 0.51 & 24 \\
\hline $\begin{array}{l}\text { Aperture spacing across } \\
\text { branch }(\mathrm{mm})\end{array}$ & 0.32 & 0.037 & 0.0014 & 0.39 & 0.25 & 26 \\
\hline $\begin{array}{l}\text { Aperture spacing along } \\
\text { branch }(\mathrm{mm})\end{array}$ & 0.28 & 0.030 & 0.0009 & 0.37 & 0.22 & 52 \\
\hline Aperture width (mm) & 0.13 & 0.010 & 0.0001 & 0.16 & 0.11 & 53 \\
\hline $\begin{array}{l}\text { Maximal chamber width } \\
\text { aside fenestrules (mm) }\end{array}$ & 0.13 & 0.015 & 0.0002 & 0.16 & 0.11 & 29 \\
\hline $\begin{array}{l}\text { Chamber length aside } \\
\text { fenestrules }(\mathrm{mm})\end{array}$ & 0.26 & 0.027 & 0.0007 & 0.31 & 0.22 & 29 \\
\hline $\begin{array}{l}\text { Maximal chamber width } \\
\text { aside dissepiments } \\
(\mathrm{mm})\end{array}$ & 0.18 & 0.016 & 0.0003 & 0.21 & 0.16 & 29 \\
\hline $\begin{array}{l}\text { Chamber length aside } \\
\text { dissepiments }(\mathrm{mm})\end{array}$ & 0.26 & 0.031 & 0.0009 & 0.35 & 0.21 & 29 \\
\hline Chamber depth (mm) & 0.18 & 0.020 & 0.0004 & 0.23 & 0.14 & 15 \\
\hline $\begin{array}{l}\text { Angle transverse } \\
\text { wall-basal plate } \\
\text { (degrees) }\end{array}$ & 62.88 & 8.11 & 65.76 & 74.17 & 50.64 & 11 \\
\hline
\end{tabular}

and low, blunt, nodes lacking. Two rows of autozooecia per branch, apertures lobulated with a thick peristome. Fenestrules moderately large, elliptical to rectangular, irregular in size.

Interior: Autozooecial chambers flask-shaped, long vestibule in lateral position. Axial wall straight, locally sinuous. Hemisepta lacking. Basal plate almost flat, granular skeleton thick in the keel. Microstyles abundant, macrostyles on the reverse of branches. Heterozooecia not observed.

Remarks. - U. parva sp. nov. differs from U. nobilis, the type species, in its more delicate meshwork and shorter zooecial chambers; from $U$. parallela [Barrande, 1894 in Počta (1894)], from the Lower Devonian of Prague Basin, in having wider branches with a longer spacing and shorter chambers. U. parva sp. nov. resembles U. vera Morozova \& Lavrentjeva, 1998, from the Emsian-Eifelian of Transcaucasia, but it has thinner branches and shorter chambers than the latter. $U$. aff. nobilis Bigey, 1972a from the Emsian of the Armorican Massif (France) is similar to $U$. parva sp. nov. in its branch width, but its branch spacing is wider and fenestrules are larger.

Occurrence. - Emsian-lower Eifelian. Arnao and Moniello Inlet, Cantabrian Zone, NW Spain.

\section{Genus Semicoscinium Prout, 1859}

Type species. - Semicoscinium rhomboideum Prout, 1859; Hamilton, Eifelian (Middle Devonian), Falls of the Ohio, Indiana, USA.

\section{Semicoscinium discretum (Prantl, 1932)}

Figure $3 \mathrm{~A}-\mathrm{G}$, Table 3

1932 Isotrypa discreta; Prantl; p. 25, pl. 4, fig. 11 (non 10), pl. 5, fig. 18.

1932 Fenestella capillosa Počta (pars). - Prantl, pl. 1, fig 11 (non pl. 2, fig. 5).

1932 Fenestella pannosa Počta (pars). - Prantl, pl. 2, fig. 9 (non pl. 1, fig. 4, pl. 2, fig. 8).

1932 Semicoscinium sacculus sacculus (Barrande) (pars). Prantl, pl. 2, fig. 18 (non pl. 2, fig. 17, pl. 3, figs 6-7).

1986 Semicoscinium discreta (Prantl, 1932). - McKinney \& Křriž, pp. 38-39, fig. 21.

1998 Semicoscinium cf. discretum (Prantl, 1932) (pars). Suárez Andrés, pp. 194-195, pl. 1, fig. 8, pl. 2, figs $1-4$.

2011 Quadrisemicoscinium discretum (Prantl, 1932). Ernst et al., pp. 325-326, figs 10g-h, 11a-c, 12a-c.

Material. - Six specimens, DGO 12831-12836.

Description. - Exterior: Reticulate zoarium; branches straight or slightly sinuous, connected by broad, oval dissepiments. Keel high, separating two rows of autozooecia. Circular apertures rimmed by a peristome. Fenestrules oval, not too long. Superstructure consisting of apical widening of keel with scalloped flanks. The ichnogenus Caupokeras McKinney, 2009 may occur on the superstructure.

Figure 3. A-G - Semicoscinium discretum (Prantl, 1932). Moniello Formation. • A - tangential section cutting festooned keel tops, DGO 12834. - B - oblique tangential section from chamber bases to lower part of keels, DGO $12835 \cdot \bullet \mathrm{C}$ - deep tangential section showing morphological variability of chambers, DGO 12836. - D-F - DGO 12835; D - deep tangential section through chamber base, basal plate and reverse macrostylets; E - longitudinal section; $\mathrm{F}$ - transverse section of a branch showing the superstructure. $\bullet \mathrm{G}$ - transverse section of a colony with tubes of Caupokeras developed on the upper part of keels, DGO 12831. • H, I - Eosemicoscinium aff. burultobense Plamenskaya, 1991. Moniello Formation, DGO 12887; H - oblique tangential section; I - transverse section of branches showing thin keels. 
Juan Luis Suárez Andrés \& Patrick N. Wyse Jackson • Lower-Middle Devonian fenestrate Bryozoa (NW Spain)
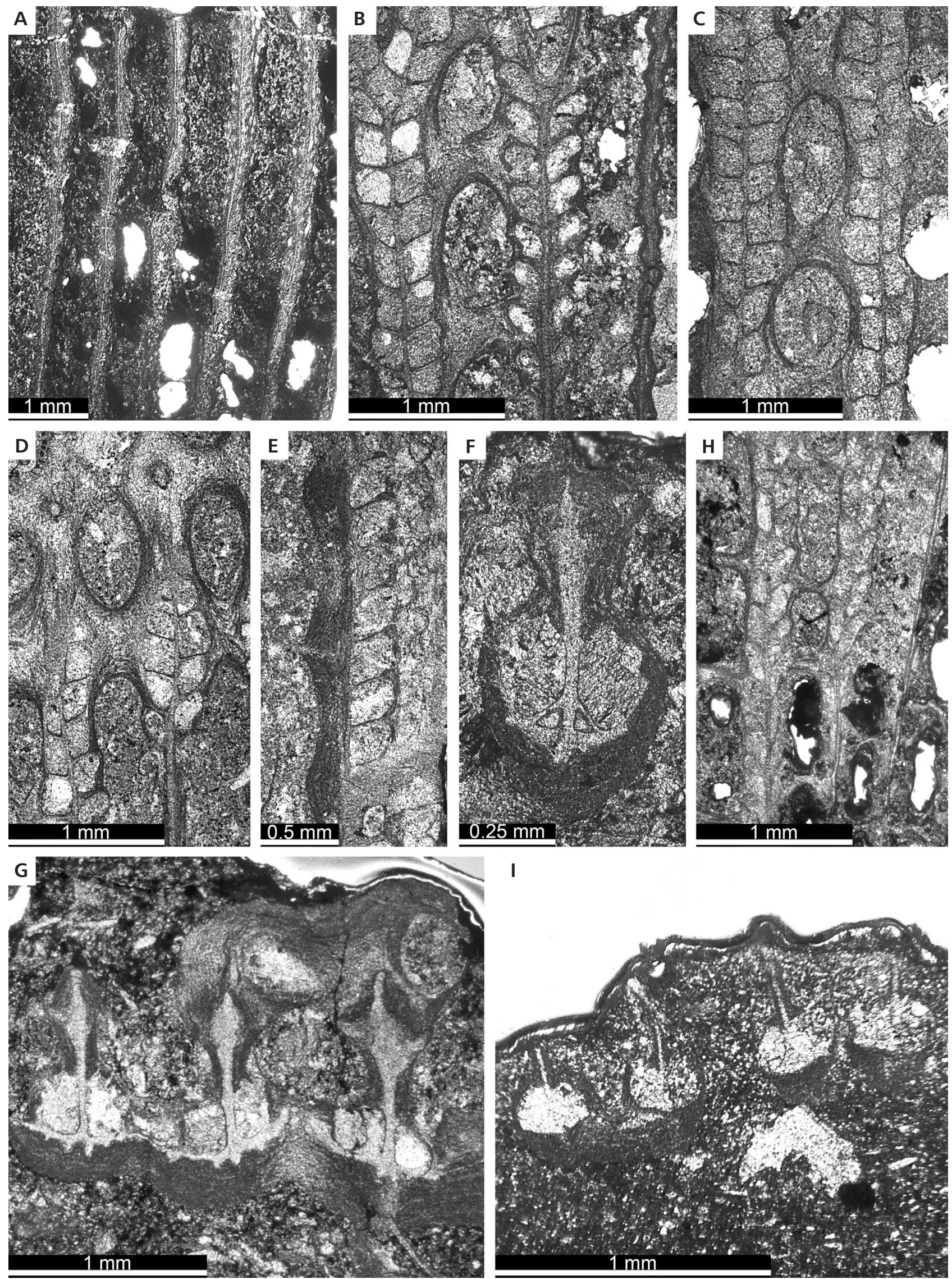
Table 3. Measurements of Semicoscinium discretum (Prantl, 1932). Abbreviations as in Table 1.

\begin{tabular}{|c|c|c|c|c|c|c|}
\hline & $\mathrm{X}$ & SD & $\mathrm{CV}$ & MAX & MIN & $\mathrm{N}$ \\
\hline Branch thickness (mm) & 0.88 & 0.125 & 0.0156 & 1.16 & 0.70 & 30 \\
\hline Branch width (mm) & 0.49 & 0.057 & 0.0032 & 0.62 & 0.40 & 26 \\
\hline Fenestrule length (mm) & 0.72 & 0.101 & 0.0102 & 0.93 & 0.56 & 28 \\
\hline Fenestrule width (mm) & 0.39 & 0.067 & 0.0045 & 0.61 & 0.26 & 29 \\
\hline Dissepiment width (mm) & 0.40 & 0.116 & 0.0135 & 0.69 & 0.24 & 33 \\
\hline $\begin{array}{l}\text { Distance between branch } \\
\text { centres }(\mathrm{mm})\end{array}$ & 0.71 & 0.135 & 0.0182 & 0.92 & 0.38 & 56 \\
\hline $\begin{array}{l}\text { Aperture spacing across } \\
\text { branch }(\mathrm{mm})\end{array}$ & 0.30 & 0.049 & 0.0024 & 0.41 & 0.23 & 22 \\
\hline $\begin{array}{l}\text { Aperture spacing along } \\
\text { branch }(\mathrm{mm})\end{array}$ & 0.27 & 0.029 & 0.0008 & 0.33 & 0.21 & 39 \\
\hline Aperture width (mm) & 0.13 & 0.026 & 0.0007 & 0.19 & 0.09 & 19 \\
\hline $\begin{array}{l}\text { Maximal chamber width } \\
\text { aside fenestrules (mm) }\end{array}$ & 0.16 & 0.021 & 0.0005 & 0.21 & 0.10 & 48 \\
\hline $\begin{array}{l}\text { Chamber length aside } \\
\text { fenestrules }(\mathrm{mm})\end{array}$ & 0.26 & 0.027 & 0.0007 & 0.34 & 0.21 & 48 \\
\hline Chamber depth & 0.28 & 0.044 & 0.0019 & 0.36 & 0.21 & 40 \\
\hline $\begin{array}{l}\text { Maximal chamber width } \\
\text { aside dissepiments (mm) }\end{array}$ & 0.23 & 0.033 & 0.0011 & 0.29 & 0.16 & 48 \\
\hline $\begin{array}{l}\text { Chamber length aside } \\
\text { dissepiments }(\mathrm{mm})\end{array}$ & 0.27 & 0.031 & 0.0010 & 0.34 & 0.22 & 48 \\
\hline $\begin{array}{l}\text { Angle transverse } \\
\text { wall-basal plate } \\
\text { (degrees) }\end{array}$ & 70.15 & 4.64 & 21.51 & 79.88 & 59.68 & 40 \\
\hline
\end{tabular}

Interior: Autozooecial chambers box-shaped, higher than long, with a short vestibule. Deep tangential section approximately square, chambers larger aside dissepiments. Axial wall straight, locally slightly sinuous. Hemisepta absent. Basal plate thick, faintly concave, with 3-5 robust longitudinal reverse ridges. Granular skeleton widened on the upper region of the keel, with a trident-shaped end. Coarse macrostyles commonly present on the reverse surface of branches; minute microstyles common. Laminar skeleton thickened on the reverse of branches, preferentially alongside dissepiments. Possible brood chambers occupy zooecial positions aside dissepiments.

Remarks. - Semicoscinium discretum differs from S. fungiforme (Plamenskaya, 1991), from the Emsian of Kazakhstan, in having wider branches and larger fenestrules.

Occurrence. - Zlíchov Limestone, Emsian, Prague Basin, Czech Republic; Santa Lucía and Moniello Formations, upper Emsian-lower Eifelian, Cantabrian Zone, NW Spain.

\section{Genus Eosemicoscinium Morozova, 1987}

Type species. - Semicoscinium pyramidatum Kopajevich, 1975; Lower Silurian, Estonia.
Table 4. Measurements of Eosemicoscinium aff. burultobense Plamenskaya, 1991. Abbreviations as in Table 1.

\begin{tabular}{|c|c|c|c|c|c|c|}
\hline & $\mathrm{X}$ & SD & $\mathrm{CV}$ & MAX & MIN & $\mathrm{N}$ \\
\hline Branch thickness (mm) & 0.64 & 0.013 & 0.0002 & 0.65 & 0.62 & 3 \\
\hline Branch width (mm) & 0.30 & 0.036 & 0.0013 & 0.35 & 0.28 & 3 \\
\hline Fenestrule length (mm) & 0.61 & 0.081 & 0.0065 & 0.70 & 0.55 & 3 \\
\hline Fenestrule width (mm) & 0.19 & 0.037 & 0.0014 & 0.23 & 0.16 & 3 \\
\hline Dissepiment width (mm) & 0.08 & 0.008 & 0.0001 & 0.09 & 0.07 & 4 \\
\hline $\begin{array}{l}\text { Distance between branch } \\
\text { centres }(\mathrm{mm})\end{array}$ & 0.44 & 0.047 & 0.0022 & 0.52 & 0.40 & 7 \\
\hline $\begin{array}{l}\text { Aperture spacing across } \\
\text { branch }(\mathrm{mm})\end{array}$ & 0.23 & 0.030 & 0.0009 & 0.28 & 0.18 & 16 \\
\hline $\begin{array}{l}\text { Aperture spacing along } \\
\text { branch }(\mathrm{mm})\end{array}$ & 0.28 & 0.030 & 0.0009 & 0.33 & 0.24 & 11 \\
\hline Aperture width (mm) & 0.10 & 0.007 & 0.0001 & 0.11 & 0.09 & 11 \\
\hline $\begin{array}{l}\text { Maximal chamber width } \\
\text { aside fenestrules (mm) }\end{array}$ & 0.10 & 0.016 & 0.0003 & 0.11 & 0.09 & 2 \\
\hline $\begin{array}{l}\text { Chamber length aside } \\
\text { fenestrules }(\mathrm{mm})\end{array}$ & 0.23 & 0.032 & 0.0011 & 0.25 & 0.20 & 2 \\
\hline
\end{tabular}

Eosemicoscinium aff. burultobense Plamenskaya, 1991 Figure $3 \mathrm{H}-\mathrm{I}$, Table 4

aff. 1991 Eosemicoscinium burultobense; Plamenskaya, pp. 103-104, pl. 20, figs 6-7.

Material. - A single fragmentary specimen, DGO 12887.

Description. - Exterior: Zoarium reticulate, branches straight and thin, connected by delicate round dissepiments, somewhat irregularly spaced. Keel very high, thin, apex spear-shaped, nodes lacking. Autozooecia arranged in two rows separated by the keel, zooecial apertures circular, alternating, almost orthogonal to the meshwork plane, with a thin, short peristome. Fenestrules rectangular, elongate, some not much longer than wide, exceptionally wider than long due to abnormally short dissepiment spacing.

Interior: Autozooecial chambers parallelogram-shaped in deep tangential section, chamber length larger than width. Kidney-shaped chamber morphology in shallower tangential sections, cutting vestibule and chamber vault. Short, distal tube with very low divergence from branch axial plane. Zooecial transversal walls forming sharp angles with axial wall, lateral walls generally straight. Hemisepta lacking. Basal plate concave, sometimes almost flat; reverse longitudinal reverse ridges few and minute or absent. Microstyles relatively coarse, arranged in irregular rows on the reverse of branches. Laminar skeleton thin on the obverse, slightly thickened on the reverse of branches. Heterozooecia not observed.

Remarks. - The specimen can be assigned with certainty to Eosemicoscinium; the meshwork is more delicate than 
those of known Devonian species, including E. serratum Ernst, 2012, identified in the Emsian La Vid Group (León, Spain), from which it differs in the absence of scalloped laminar skeleton on the keel. The morphology of the studied specimen resembles that of E. burultobense Plamenskaya, 1991, from the Emsian of Kazakhstan, in the spacing of apertures in a single row. Nevertheless, branch width and spacing are slightly smaller in the Spanish specimen, so it has been kept in open nomenclature.

Occurrence. - Upper Emsian-Eifelian. Moniello Inlet, Cantabrian Zone, NW Spain.

\section{Genus Bigeyina Suárez Andrés \& McKinney, 2010}

Type species. - Cyclopelta winteri Bornemann, 1884; Eifelian (Middle Devonian), Germany.

\section{Bigeyina ibera Ernst, 2012}

Figure 4A-E, Table 5

2012 Bigeyina ibera; Ernst, p. 235, fig. 19a-g.

Material. - Nine specimens, DGO 12934-12940, 13035-13036.

Description. - Ex teri or: Zoarium reticulate, narrow conical, occasionally tubular, individual or composite. Obverse on the outer surface. Branches straight or slightly sinuous, dissepiments robust, forming rings on the reverse surface, with wing-shaped cross section. Keel high, supporting superstructure and separating two rows of autozooecia; apertures lobulated, with a short, thin peristome. Fenestrules oval, medium sized. Superstructure an asymmetric "Y"-shaped expansion of the upper region of the keel, with regularly spaced bridges between adjacent keels.

Interior: Autozooecial chambers rounded polygonal aside dissepiments, pentagonal-rectangular aside fenestrules. Axial wall zigzag. Hemisepta absent. Basal plate concave, flattened at dissepiment junctions, without reverse ridges. Asymmetric bifurcation of granular skeleton in the superstructure. Laminar skeleton thickening on the top concave surface of keel laths may form convex deposits during astogeny. Vesicular tissue abundant. Microstyles very thin, common; macrostyles absent. Heterozooecia not observed.

Remarks. - B. ibera can be distinguished from other species present in the Moniello Formation by its colony shape and the presence of bridges between keels. Furthermore, it differs from $B$. cantabrica in having narrower branches and smaller fenestrules; from $B$. victrola in the straight morphology of branches, and from B. spinosa in having
Table 5. Measurements of Bigeyina ibera Ernst, 2012. Abbreviations as in Table 1.

\begin{tabular}{|c|c|c|c|c|c|c|}
\hline & $\mathrm{X}$ & SD & $\mathrm{CV}$ & MAX & MIN & $\mathrm{N}$ \\
\hline Branch thickness (mm) & 0.62 & 0.172 & 0.0295 & 0.94 & 0.23 & 25 \\
\hline Branch width (mm) & 0.29 & 0.088 & 0.0077 & 0.50 & 0.20 & 21 \\
\hline Fenestrule length (mm) & 0.53 & 0.057 & 0.0033 & 0.65 & 0.48 & 9 \\
\hline Fenestrule width (mm) & 0.27 & 0.045 & 0.0021 & 0.33 & 0.19 & 11 \\
\hline Dissepiment width (mm) & 0.32 & 0.076 & 0.0057 & 0.41 & 0.23 & 7 \\
\hline $\begin{array}{l}\text { Distance between branch } \\
\text { centres }(\mathrm{mm})\end{array}$ & 0.58 & 0.137 & 0.0189 & 0.88 & 0.37 & 37 \\
\hline $\begin{array}{l}\text { Aperture spacing across } \\
\text { branch (mm) }\end{array}$ & 0.30 & 0.049 & 0.0024 & 0.38 & 0.23 & 14 \\
\hline $\begin{array}{l}\text { Aperture spacing along } \\
\text { branch (mm) }\end{array}$ & 0.32 & 0.056 & 0.0032 & 0.42 & 0.25 & 28 \\
\hline Aperture width (mm) & 0.13 & 0.025 & 0.0006 & 0.17 & 0.08 & 32 \\
\hline $\begin{array}{l}\text { Maximal chamber width } \\
\text { aside fenestrules (mm) }\end{array}$ & 0.16 & 0.041 & 0.0017 & 0.24 & 0.10 & 30 \\
\hline $\begin{array}{l}\text { Chamber length aside } \\
\text { fenestrules }(\mathrm{mm})\end{array}$ & 0.24 & 0.024 & 0.0006 & 0.29 & 0.20 & 28 \\
\hline $\begin{array}{l}\text { Maximal chamber width } \\
\text { aside dissepiments (mm) }\end{array}$ & 0.21 & 0.026 & 0.0007 & 0.25 & 0.17 & 20 \\
\hline $\begin{array}{l}\text { Chamber length aside } \\
\text { dissepiments (mm) }\end{array}$ & 0.25 & 0.024 & 0.0006 & 0.28 & 0.17 & 20 \\
\hline $\begin{array}{l}\text { Angle transverse } \\
\text { wall-basal plate } \\
\text { (degrees) }\end{array}$ & 71.90 & - & - & 71.90 & 71.90 & 1 \\
\hline
\end{tabular}

more elongate chambers and in the absence of spines on the reverse surface of dissepiments. Some colonies of $B$. ibera developed complex tubular morphologies by bifurcation, as described by Suárez Andrés \& McKinney (2010), an extremely rare growth habit among fenestrates, which in this genus has only been observed in the Devonian of Spain.

Occurrence. - Emsian-lower Eifelian. Arnao and Moniello Inlet, Cantabrian Zone, NW Spain.

\section{Bigeyina victrola (McKinney \& Kříž, 1986)}

Figure 4F-I, Table 6

1986 Cyclopelta victrola; McKinney \& Kříž, p. 46, figs 25,26 .

Material. - Five specimens, DGO 12956-12960.

Description. - Exterior: Zoarium reticulate, conical. Branches sinuous, dissepiments very short and wide. Keel high, sinuous, separating two rows of autozooecia. Apertures circular, faintly lobulated, with a thin peristome. Fenestrules oval, arranged in a rhombic pattern, superstructure an expansion of the uppermost region of the keel.

Interior: Autozooecial chambers polygonal, rounded and larger close to dissepiments. Axial wall zigzag. Hemisepta 
Table 6. Measurements of Bigeyina victrola (McKinney \& Kř́̌ž, 1986). Abbreviations as in Table 1.

\begin{tabular}{|c|c|c|c|c|c|c|}
\hline & $\mathrm{X}$ & SD & $\mathrm{CV}$ & MAX & MIN & $\mathrm{N}$ \\
\hline ranch thickness (mm) & 0.83 & 0.074 & 0.0055 & 0.99 & 0.73 & 15 \\
\hline Branch width (mm) & 0.44 & 0.063 & 0.0040 & 0.55 & 0.33 & 20 \\
\hline Fenestrule length (mm) & 0.63 & 0.094 & 0.0088 & 0.84 & 0.46 & 42 \\
\hline Fenestrule width (mm) & 0.34 & 0.060 & 0.0036 & 0.51 & 0.24 & 38 \\
\hline Dissepiment width (mm) & 0.47 & 0.085 & 0.0072 & 0.73 & 0.33 & 40 \\
\hline $\begin{array}{l}\text { Aperture spacing across } \\
\text { branch }(\mathrm{mm})\end{array}$ & 0.29 & 0.042 & 0.0017 & 0.41 & 0.22 & 36 \\
\hline $\begin{array}{l}\text { Aperture spacing along } \\
\text { branch }(\mathrm{mm})\end{array}$ & 0.23 & 0.022 & 0.0005 & 0.27 & 0.18 & 42 \\
\hline Aperture width (mm) & 0.10 & 0.009 & 0.0001 & 0.12 & 0.08 & 45 \\
\hline $\begin{array}{l}\text { Maximal chamber width } \\
\text { aside fenestrules (mm) }\end{array}$ & 0.14 & 0.018 & 0.0003 & 0.18 & 0.10 & 51 \\
\hline $\begin{array}{l}\text { Chamber length aside } \\
\text { fenestrules }(\mathrm{mm})\end{array}$ & 0.21 & 0.020 & 0.0004 & 0.27 & 0.17 & 51 \\
\hline $\begin{array}{l}\text { Maximal chamber width } \\
\text { aside dissepiments }(\mathrm{mm})\end{array}$ & 0.22 & 0.031 & 0.0009 & 0.29 & 0.16 & 54 \\
\hline $\begin{array}{l}\text { Chamber length aside } \\
\text { dissepiments }(\mathrm{mm})\end{array}$ & 0.22 & 0.023 & 0.0006 & 0.27 & 0.17 & 54 \\
\hline Chamber depth (mm) & 0.23 & 0.027 & 0.0007 & 0.27 & 0.20 & 12 \\
\hline $\begin{array}{l}\text { Angle transverse } \\
\text { wall-basal plate } \\
\text { (degrees) }\end{array}$ & 7.34 & 6.25 & 39.04 & 8.46 & 6.23 & 12 \\
\hline $\begin{array}{l}\text { Distance between branch } \\
\text { centres aside fenestrules } \\
(\mathrm{mm})\end{array}$ & 0.68 & 0.066 & 0.0044 & 0.83 & 0.54 & 40 \\
\hline $\begin{array}{l}\text { Distance between branch } \\
\text { centres aside } \\
\text { dissepiments }(\mathrm{mm})\end{array}$ & 0.49 & 0.074 & 0.0055 & 0.74 & 0.37 & 38 \\
\hline
\end{tabular}

absent. Basal plate slightly concave, flattened at dissepiment junctions. Symmetric bifurcation of granular core of superstructure, laminar skeleton thickened on top of the keel laths and reverse surface of branches. Microstyles and macrostyles present on reverse surface. Heterozooecia not observed.

Remarks. - B. victrola differs from all other known species of Bigeyina in its branch sinuosity, which in turn affects the arrangement of fenestrules and of dissepiments. The characteristic annular processes on the reverse surface of the colony corresponding with dissepiments as developed in $B$. spinosa are lacking in this species.

Occurrence. - Pragian, Prague Basin, Czech Republic;
Table 7. Measurements of Bigeyina cantabrica sp. nov. Abbreviations as in Table 1.

\begin{tabular}{|c|c|c|c|c|c|c|}
\hline & $\mathrm{X}$ & SD & $\mathrm{CV}$ & MAX & MIN & $\mathrm{N}$ \\
\hline Branch thickness (mm) & 0.74 & 0.185 & 0.0343 & 1.09 & 0.28 & 65 \\
\hline Branch width (mm) & 0.38 & 0.092 & 0.0085 & 0.59 & 0.26 & 61 \\
\hline Fenestrule length (mm) & 0.63 & 0.101 & 0.0102 & 0.94 & 0.39 & 66 \\
\hline Fenestrule width (mm) & 0.32 & 0.076 & 0.0058 & 0.61 & 0.20 & 72 \\
\hline Dissepiment width (mm) & 0.57 & 0.152 & 0.0231 & 1.07 & 0.27 & 71 \\
\hline $\begin{array}{l}\text { Distance between branch } \\
\text { centres }(\mathrm{mm})\end{array}$ & 0.60 & 0.079 & 0.0063 & 0.83 & 0.34 & 106 \\
\hline cing across & 0.31 & 0.040 & 0.0016 & 0.39 & 0.22 & 104 \\
\hline $\begin{array}{l}\text { Aperture spacing along } \\
\text { branch }(\mathrm{mm})\end{array}$ & 0.27 & 0.022 & 0.0005 & 0.32 & 0.21 & 122 \\
\hline Aperture width (mm) & 0.11 & 0.011 & 0.0001 & 0.14 & 0.09 & 137 \\
\hline $\begin{array}{l}\text { ber width } \\
\text { es }(\mathrm{mm})\end{array}$ & 0.16 & 0.042 & 0.0018 & 0.29 & 0.08 & 125 \\
\hline Chamber length aside & 0.24 & 0.027 & 0.0 & 0.31 & 0.16 & 125 \\
\hline $\begin{array}{l}\text { Maximal chamber width } \\
\text { aside dissepiments (mm) }\end{array}$ & 0.24 & 0.033 & 0.0011 & 0.30 & 0.16 & 126 \\
\hline $\begin{array}{l}\text { Chamber length aside } \\
\text { dissepiments }(\mathrm{mm})\end{array}$ & 0.25 & 0.024 & 0.0006 & 0.30 & 0.19 & 126 \\
\hline Chamber depth (mm) & 0.23 & 0.033 & 0.0011 & 0.31 & 0.17 & 47 \\
\hline $\begin{array}{l}\text { Angle transverse } \\
\text { wall-basal plate } \\
\text { (degrees) }\end{array}$ & 76.95 & 8.78 & 77.13 & 89.10 & 59.38 & 47 \\
\hline
\end{tabular}

Emsian-lower Eifelian, Arnao and Moniello Inlet, Cantabrian Zone, NW Spain.

\section{Bigeyina cantabrica sp. nov.}

Figure 5A-D, Table 7

Holotype. - DGO 12860.

Paratypes. - DGO 12861-12867.

Type horizon and locality. - Upper Emsian, Moniello Formation. Arnao.

Material. - Twelve specimens, DGO 12860-12870, 12886.

Etymology. - From the Cantabrian Zone, the tectonostratigraphic unit in which the species has been identified. Gender feminine.

Figure 4. A-E - Bigeyina ibera Ernst, 2012. Moniello Formation. • A - deep tangential section showing chamber morphology, DGO 12935. • B - very shallow tangential section cutting bridged keels in uppermost superstructure, DGO 12937. $\bullet$ C - longitudinal section between two branches through dissepiments (left) and superstructure (right), DGO 12938. • D - longitudinal section of a branch, DGO 12937 . $\bullet$ E - transverse section of several branches showing asymmetrical keel laths, DGO 12937. • F-I - Bigeyina victrola (McKinney \& Kříž, 1986). Moniello Formation; F - longitudinal section of a branch, DGO $12960 . \bullet \mathrm{G}$ - transverse section of branches with one kenozooecium (arrow), DGO 12958. • H, I-tangential sections, DGO 12957; H - deep tangential section; I - shallow tangential section cutting macrostylets on the reverse of branches. 
Juan Luis Suárez Andrés \& Patrick N. Wyse Jackson • Lower-Middle Devonian fenestrate Bryozoa (NW Spain)
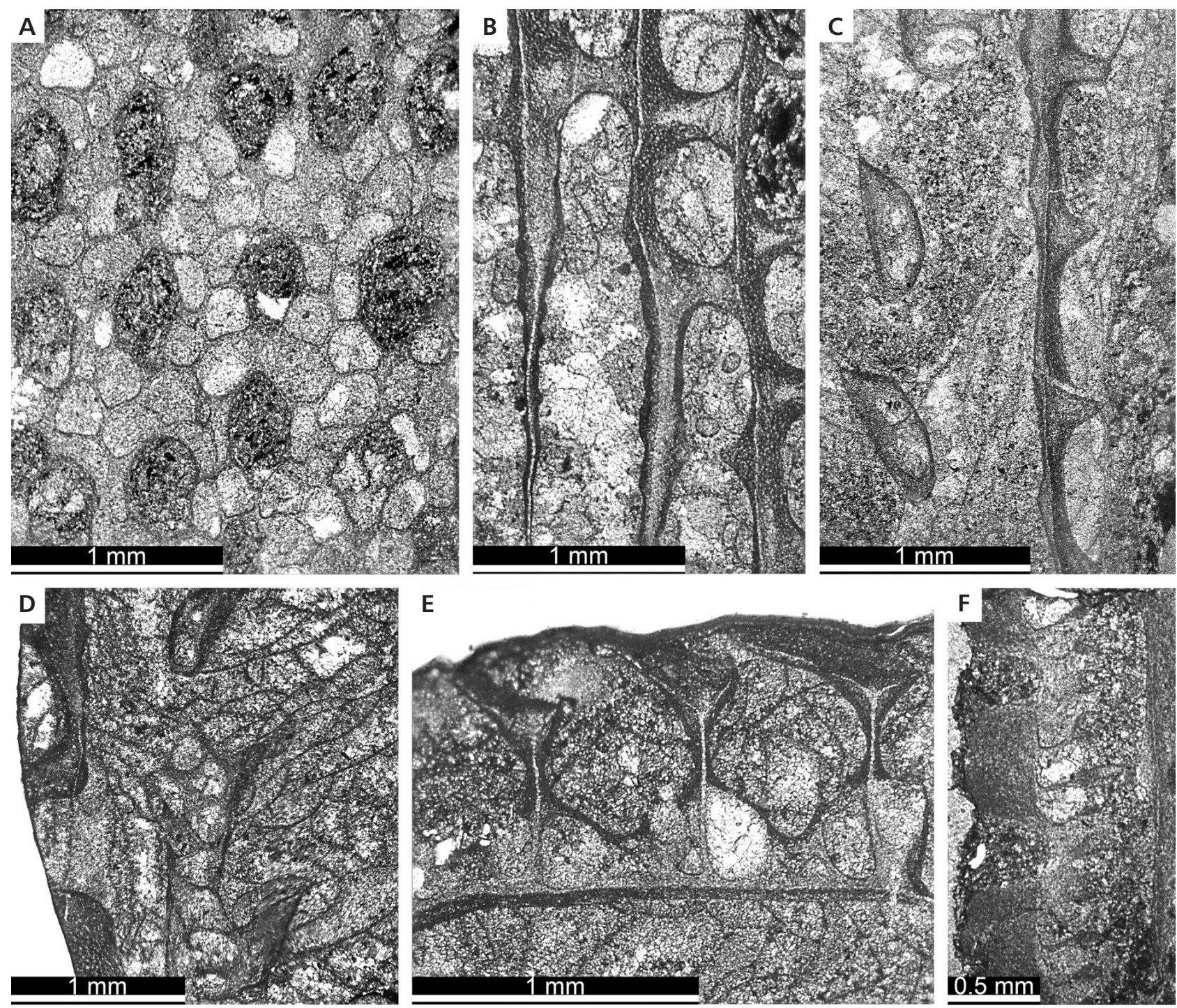

E
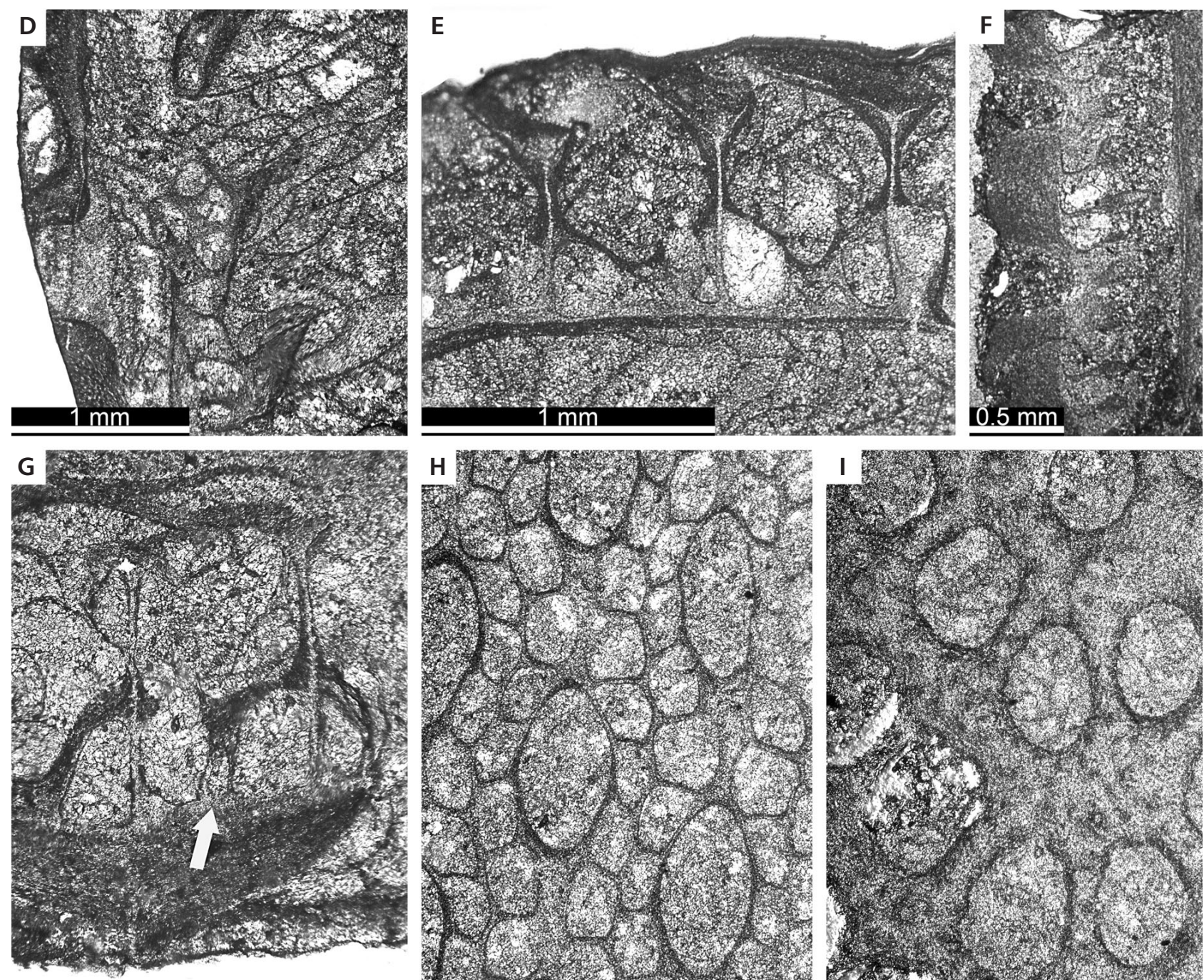

$0.5 \mathrm{~mm}$
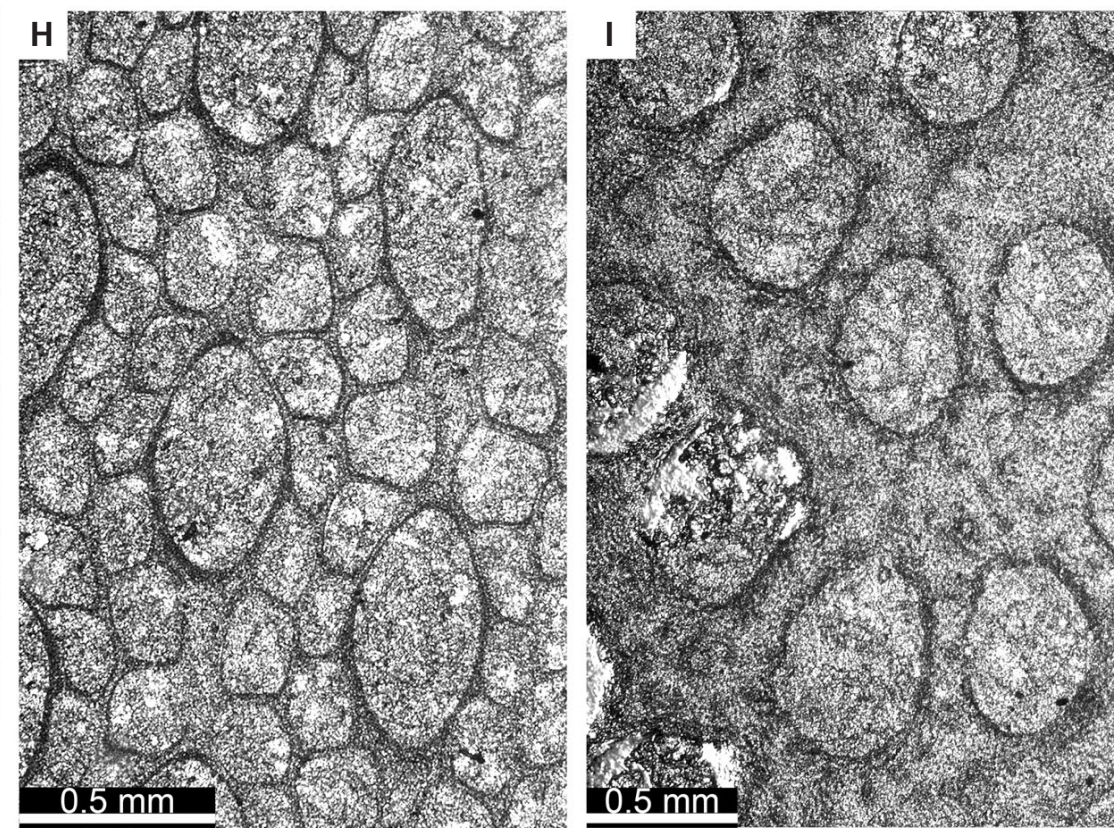
Table 8. Measurements of Bigeyina spinosa sp. nov. Abbreviations as in Table 1.

\begin{tabular}{|c|c|c|c|c|c|c|}
\hline & $\mathrm{X}$ & SD & $\mathrm{CV}$ & MAX & MIN & $\mathrm{N}$ \\
\hline Branch thickness (mm) & 0.92 & 0.114 & 0.0129 & 1.05 & 0.75 & 10 \\
\hline Branch width (mm) & 0.43 & 0.090 & 0.0081 & 0.58 & 0.31 & 9 \\
\hline Fenestrule length (mm) & 0.61 & 0.092 & 0.0084 & 0.85 & 0.45 & 29 \\
\hline Fenestrule width (mm) & 0.31 & 0.044 & 0.0019 & 0.40 & 0.22 & 31 \\
\hline Dissepiment width (mm) & 0.44 & 0.090 & 0.0081 & 0.58 & 0.25 & 23 \\
\hline $\begin{array}{l}\text { Distance between branch } \\
\text { centres }(\mathrm{mm})\end{array}$ & 0.60 & 0.093 & 0.0086 & 0.88 & 0.41 & 29 \\
\hline $\begin{array}{l}\text { Aperture spacing across } \\
\text { branch }(\mathrm{mm})\end{array}$ & 0.29 & 0.049 & 0.0024 & 0.40 & 0.21 & 28 \\
\hline $\begin{array}{l}\text { Aperture spacing along } \\
\text { branch }(\mathrm{mm})\end{array}$ & 0.24 & 0.022 & 0.0005 & 0.30 & 0.19 & 40 \\
\hline Aperture width (mm) & 0.10 & 0.010 & 0.0001 & 0.12 & 0.08 & 41 \\
\hline $\begin{array}{l}\text { Maximal chamber width } \\
\text { aside fenestrules (mm) }\end{array}$ & 0.14 & 0.017 & 0.0003 & 0.18 & 0.11 & 30 \\
\hline $\begin{array}{l}\text { Chamber length aside } \\
\text { fenestrules }(\mathrm{mm})\end{array}$ & 0.22 & 0.025 & 0.0006 & 0.30 & 0.18 & 30 \\
\hline $\begin{array}{l}\text { Maximal chamber width } \\
\text { aside dissepiments }(\mathrm{mm})\end{array}$ & 0.23 & 0.022 & 0.0005 & 0.28 & 0.19 & 33 \\
\hline $\begin{array}{l}\text { Chamber length aside } \\
\text { dissepiments }(\mathrm{mm})\end{array}$ & 0.22 & 0.038 & 0.0014 & 0.31 & 0.14 & 33 \\
\hline Chamber depth (mm) & 0.23 & 0.023 & 0.0005 & 0.27 & 0.20 & 7 \\
\hline $\begin{array}{l}\text { Angle transverse } \\
\text { wall-basal plate } \\
\text { (degrees) }\end{array}$ & 70.26 & 6.39 & 40.83 & 79.30 & 60.60 & 7 \\
\hline
\end{tabular}

Diagnosis. - Bigeyina with straight branches, symmetrically bifurcated keel and a single longitudinal ridge on the reverse surface of the basal plate.

Description. - Exterior: Zoarium reticulate, moderately open conical, obverse on the outer surface. Branches straight, dissepiments short and wide, transversely aligned, forming annular projections on the inner surface of the cone. Keel high, supporting the superstructure. Autozooecia arranged in two rows, apertures circular, possibly lobulated, peristome thin. Fenestrules oval, medium sized, regularly arranged. Superstructure an apical symmetrical expansion of the keel.

Interior: Autozooecial chambers bimodal; rounded pentagonal or hexagonal in dissepiment junctions, smaller and elongate aside fenestrules. Axial wall zigzag, occasionally almost straight. Hemisepta absent. Basal plate concave with a single reverse ridge between dissepiments, almost flat and lacking ridges in dissepiment junctions. Granular skeleton bifurcated in the keel top. Astogenetic thickening of the laminar skeleton on the upper surface of the superstructure. Microstyles minute, very common; macrostyles absent. Heterozooecia not observed.

Remarks. - B. cantabrica sp. nov. differs from B. ibera in the colony shape and absence of keel bridges. From B. spinosa it differs in the absence of spines on the reverse surface of dissepiments and the presence of a single reverse ridge in the basal plate.

Occurrence. - Emsian-lower Eifelian. Arnao and Moniello Inlet, Cantabrian Zone, NW Spain.

\section{Bigeyina spinosa sp. nov.}

Figure 5E-H, Table 8

Holotype. - DGO 12857.

Paratypes. - DGO 12967.

Type horizon and locality. - Upper Emsian, Moniello Formation, Arnao.

Material. - Five specimens, DGO 12857-12859, 12890, 12967.

Etymology. - From Latin, spinosa, referring to the presence of spines on the distal edge of the reverse annular projections of dissepiments. Gender feminine.

Diagnosis. - Bigeyina with straight branches and spines on the reverse of the colony, placed on distal edges of annular dissepiments.

Description. - Exterior: Zoarium reticulate, conical, obverse on the outer surface. Branches straight, dissepiments short and wide, aligned transversely to branches and forming annular projections on the reverse of the colony. Small, robust spines on the reverse, placed on the distal edge of dissepiments and pointing distally. Keel high, separating two rows of autozooecia; zooecial apertures lobulated, peristome thin and short. Fenestrules oval, medium sized, regularly arranged. Superstructure a widened lath on

Figure 5. A-D - Bigeyina cantabrica sp. nov. Moniello Formation. • A - longitudinal section of a branch, DGO 12870 . $\bullet$ B - deep tangential section, DGO 12863. $\bullet \mathrm{C}$ - shallow tangential section of the reverse of branches, cutting the longitudinal ridge under the basal plate and abundant microstylets, DGO 12863. • D - transverse section of several branches between dissepiments showing the ridged basal plate (arrow), DGO 12870 . $\bullet$ E-H - Bigeyina spinosa sp. nov. Moniello Formation. $\bullet \mathrm{E}-\mathrm{G}$ - holotype, DGO 12857; E - longitudinal section of a branch cutting zooecial chambers and Caupokeras (arrows); F - shallow tangential section of the reverse of the colony showing spines placed at distal edges of dissepiments over fenestrules (arrows); $\mathrm{G}$ - deep tangential section. $\bullet \mathrm{H}$ - transverse section of a branch; microstructural details and Caupokeras can be observed, DGO 12890 . 
Juan Luis Suárez Andrés \& Patrick N. Wyse Jackson • Lower-Middle Devonian fenestrate Bryozoa (NW Spain)
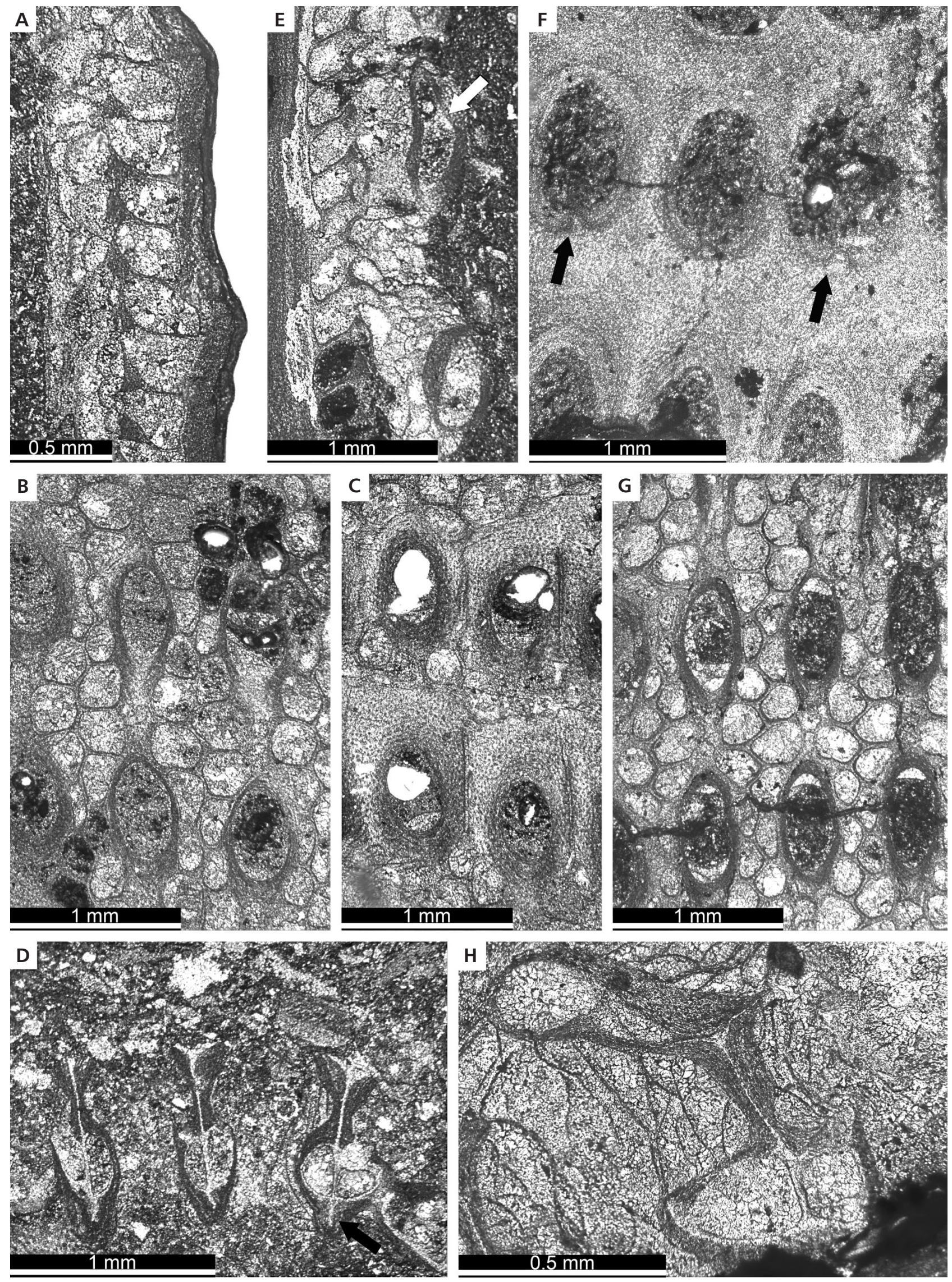
Table 9. Measurements of Fenestrapora elegans sp. nov. Abbreviations as in Table 1.

\begin{tabular}{|c|c|c|c|c|c|c|}
\hline & $\mathrm{X}$ & SD & $\mathrm{CV}$ & MAX & MIN & $\mathrm{N}$ \\
\hline Branch thickness (mm) & 1.22 & 0.230 & 0.0530 & 1.53 & 0.95 & 13 \\
\hline Branch width (mm) & 0.56 & 0.073 & 0.0053 & 0.66 & 0.43 & 14 \\
\hline Fenestrule length (mm) & 1.09 & 0.140 & 0.0195 & 1.33 & 0.75 & 19 \\
\hline Fenestrule width (mm) & 0.50 & 0.105 & 0.0111 & 0.69 & 0.30 & 19 \\
\hline Dissepiment width (mm) & 0.65 & 0.123 & 0.0152 & 0.86 & 0.37 & 18 \\
\hline $\begin{array}{l}\text { Distance between branch } \\
\text { centres aside fenestrules } \\
(\mathrm{mm})\end{array}$ & 0.96 & 0.132 & 0.0174 & 1.24 & 0.74 & 15 \\
\hline $\begin{array}{l}\text { Distance between branch } \\
\text { centres aside } \\
\text { dissepiments }(\mathrm{mm})\end{array}$ & 0.74 & 0.109 & 0.0119 & 0.96 & 0.55 & 16 \\
\hline icing across & 0.31 & 0.041 & 0.0017 & 0.42 & 0.26 & 20 \\
\hline $\begin{array}{l}\text { Aperture spacing along } \\
\text { branch }(\mathrm{mm})\end{array}$ & 0.28 & 0.022 & 05 & 0.33 & 0.25 & 30 \\
\hline Aperture width (mm) & 0.13 & 0.023 & 0.0005 & 0.20 & 0.11 & 19 \\
\hline $\begin{array}{l}\text { Maximal chamber width } \\
\text { aside fenestrules (mm) }\end{array}$ & 0.16 & 0.013 & 002 & 0.19 & 0.14 & 35 \\
\hline $\begin{array}{l}\text { Chamber length aside } \\
\text { fenestrules }(\mathrm{mm})\end{array}$ & 0.25 & 0.024 & 0.0006 & 0.32 & 0.22 & 35 \\
\hline $\begin{array}{l}\text { Maximal chamber width } \\
\text { aside dissepiments (mm) }\end{array}$ & 0.27 & 0.038 & 0.0014 & 0.31 & 0.19 & 22 \\
\hline $\begin{array}{l}\text { Chamber length aside } \\
\text { dissepiments }(\mathrm{mm})\end{array}$ & 0.27 & 0.024 & 0.0006 & 0.32 & 0.22 & 22 \\
\hline Chamber depth (mm) & 0.33 & 0.037 & 0.0013 & 0.38 & 0.24 & 16 \\
\hline $\begin{array}{l}\text { Angle transverse } \\
\text { wall-basal plate } \\
\text { (degrees) }\end{array}$ & 70.83 & 9.08 & 82.40 & 89.48 & 60.14 & 19 \\
\hline
\end{tabular}

top of the keels. In some specimens the ichnogenus Caupokeras McKinney, 2009 is developed.

Interior: Autozooecial chambers large, equidimensional, pentagonal or hexagonal at dissepiment junctions; smaller and elongate pentagonal aside fenestrules. Axial wall zigzag, hemisepta absent. Basal plate concave, flattened at dissepiment junctions. Granular skeleton of the superstructure forming a very open, symmetrical bifurcation. Microstyles numerous, very thin. Heterozooecia not observed.

Remarks. - B. spinosa sp. nov. differs from all described Bigeyina species in having small spines on the reverse of the colony, developed on the distal edge of annular projections of dissepiments.

Occurrence. - Emsian-lower Eifelian, Arnao and Moniello Inlet, Cantabrian Zone, NW Spain.

\section{Genus Fenestrapora Hall, 1885}

Type species. - Fenestrapora biperforata Hall, 1885; Middle Devonian, New York, USA.

Fenestrapora elegans sp. nov.

Figure 6A-E, Table 9

Holotype. - DGO 12808.

Paratypes. - DGO 12809-12811.

Type horizon and locality. - Upper Emsian-lower Eifelian, Moniello Formation, Arnao.

Material. - Four specimens, DGO 12808-12811.

Etymology. - From Latin elegans, elegant. Gender feminine.

Diagnosis. - Fenestrapora with strongly sinuous branches, sharp spear-shaped keels, aviculomorphs on dissepiments.

Description. - Exterior: Zoarium reticulate, unknown if conical or fan-shaped. Branches sinuous, moderately wide, dissepiments short and wide. Keel high and thin, with a sharp edge. Two rows of autozooecia per branch, apertures rounded, with peristome. Fenestrules elliptical, moderately large, arranged in a rhombic pattern.

Interior: Autozooecial chambers rectangular, larger at dissepiment junctions, with a pore in the proximal region of the lateral wall connecting the chamber with the outer surface. Axial wall straight, occasionally sinuous. Hemisepta lacking. Basal plate concave, thick, laminar skeleton well developed, pierced by thin microstyles. Possible reproductive heterozooecia with enlarged chambers occupying positions aside dissepiments. Aviculomorphs present on the obverse of dissepiments, size roughly equal to autozooecia.

Figure 6. A-E - Fenestrapora elegans sp. nov. Moniello Formation. • A - longitudinal section, holotype, DGO 12808 . • B-E - DGO 12809; B - shallow tangential section showing branch sinuosity; C - tangential section, aviculomorphs on dissepiments (arrows); D - transverse section of a branch showing microstructural details; E - tangential section; minute pores placed in the proximal region of autozooecial chambers (arrows). - F-J - Fenestrapora occidentalis Ulrich, 1890 , Moniello Formation. $\bullet$ F - longitudinal section of a branch, DGO 12847 . $\bullet$ G - oblique tangential section showing morphological differences in axial walls and chambers of neighbouring branches, DGO 12853 . $\bullet H-$ transverse section of two branches with heavily thickened laminar skeleton; lateral nodes of scalloped superstructure can be observed (arrow), DGO 12842 . $\bullet$ I - tangential section of the reverse cutting abundant styles and aviculomorphs (arrows). DGO 12846. • J - deep tangential section, DGO 12843. 
Juan Luis Suárez Andrés \& Patrick N. Wyse Jackson • Lower-Middle Devonian fenestrate Bryozoa (NW Spain)
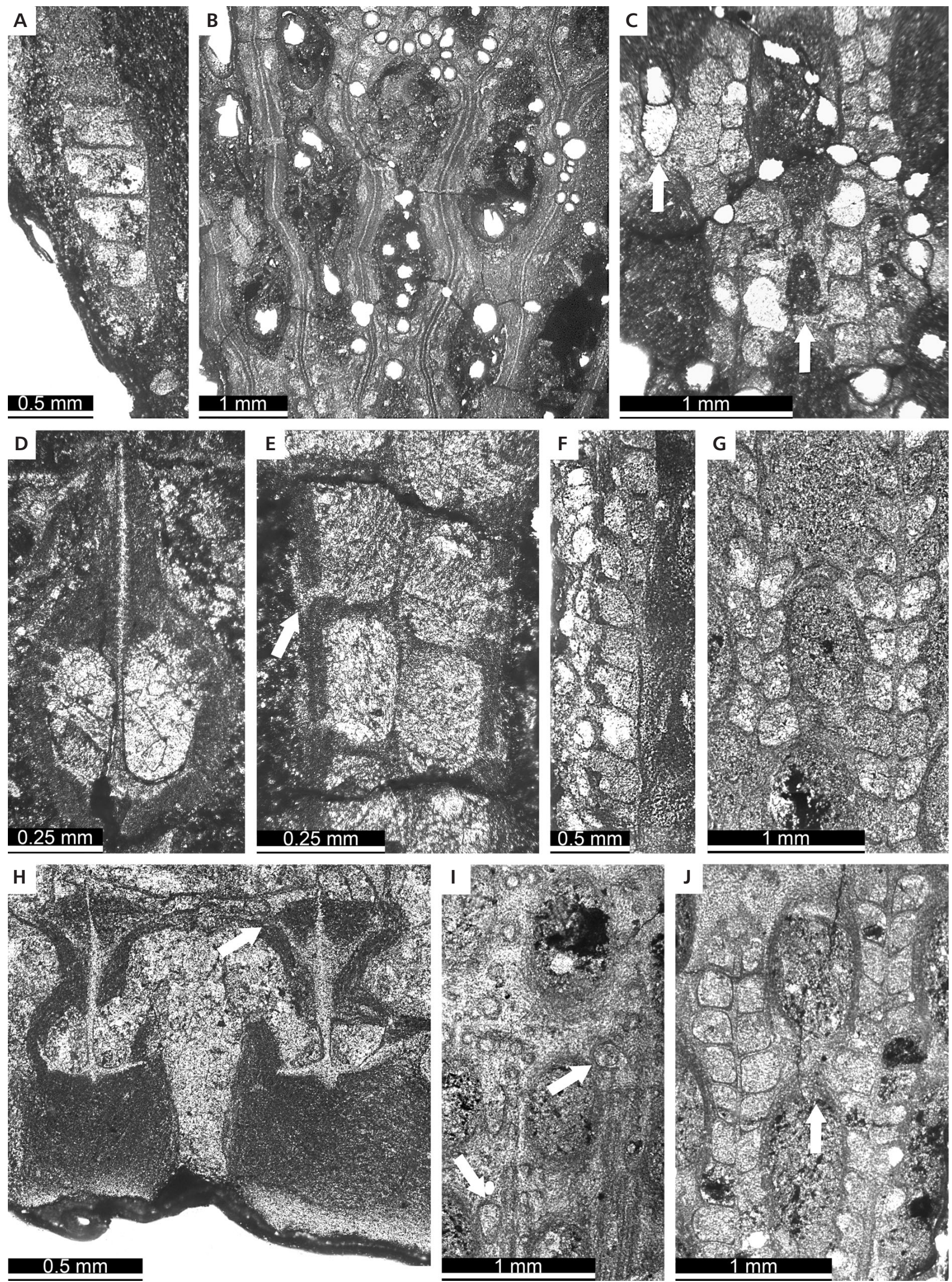
Table 10. Measurements of Fenestrapora occidentalis Ulrich, 1890. Abbreviations as in Table 1.

\begin{tabular}{|c|c|c|c|c|c|c|}
\hline & $\mathrm{X}$ & SD & $\mathrm{CV}$ & MAX & MIN & $\mathrm{N}$ \\
\hline Branch thickness (mm) & 1.00 & 0.201 & 0.0404 & 1.42 & 0.74 & 74 \\
\hline Branch width (mm) & 0.47 & 0.076 & 0.0058 & 0.70 & 0.39 & 83 \\
\hline Fenestrule length (mm) & 0.70 & 0.078 & 0.0061 & 0.83 & 0.56 & 102 \\
\hline Fenestrule width (mm) & 0.38 & 0.068 & 0.0047 & 0.50 & 0.26 & 98 \\
\hline Dissepiment width (mm) & 0.37 & 0.058 & 0.0033 & 0.47 & 0.29 & 128 \\
\hline $\begin{array}{l}\text { Distance between branch } \\
\text { centres }(\mathrm{mm})\end{array}$ & 0.77 & 0.112 & 0.0125 & 0.95 & 0.54 & 140 \\
\hline $\begin{array}{l}\text { Aperture spacing across } \\
\text { branch }(\mathrm{mm})\end{array}$ & 0.30 & 0.025 & 0.0006 & 0.35 & 0.27 & 26 \\
\hline $\begin{array}{l}\text { Aperture spacing along } \\
\text { branch }(\mathrm{mm})\end{array}$ & 0.27 & 0.018 & 0.0003 & 0.30 & 0.25 & 54 \\
\hline Aperture width (mm) & 0.12 & 0.014 & 0.0002 & 0.15 & 0.10 & 38 \\
\hline $\begin{array}{l}\text { Maximal chamber width } \\
\text { aside fenestrules (mm) }\end{array}$ & 0.15 & 0.018 & 0.0003 & 0.19 & 0.13 & 209 \\
\hline $\begin{array}{l}\text { Chamber length aside } \\
\text { fenestrules }(\mathrm{mm})\end{array}$ & 0.25 & 0.024 & 0.0006 & 0.30 & 0.22 & 209 \\
\hline $\begin{array}{l}\text { Maximal chamber width } \\
\text { aside dissepiments }(\mathrm{mm})\end{array}$ & 0.23 & 0.018 & 0.0003 & 0.26 & 0.17 & 193 \\
\hline $\begin{array}{l}\text { Chamber length aside } \\
\text { dissepiments }(\mathrm{mm})\end{array}$ & 0.26 & 0.031 & 0.0010 & 0.32 & 0.21 & 193 \\
\hline Chamber depth (mm) & 0.26 & 0.031 & 0.0009 & 0.31 & 0.22 & 50 \\
\hline $\begin{array}{l}\text { Angle transverse } \\
\text { wall-basal plate } \\
\text { (degrees) }\end{array}$ & 69.32 & 5.85 & 34.21 & 77.64 & 61.53 & 44 \\
\hline
\end{tabular}

Remarks. $-F$. elegans sp. nov. differs from all known species of Fenestrapora in the shape of branches and keels, as well as the presence of pores in zooecial chambers. This character, present in some Semicosciniidae, has been previously observed also in Septatopora Engel, 1975, Polypora stenostoma Tavener-Smith, 1971, Dissotrypa Ernst \& Königshof, 2010 and Adlatipora Gautier et al., 2013, though pores in these taxa are placed on the frontal wall instead of the lateral ones.

Occurrence. - Upper Emsian-lower Eifelian, Arnao and Moniello Inlet, Cantabrian Zone, NW Spain.

\section{Fenestrapora occidentalis Ulrich, 1890}

Figure 6F-J, Table 10

1890 Fenestrapora occidentalis; Ulrich, pp. 558-559, pl. 44, figs 2, 2a, pl. 54, figs 7-7e.

1998 Semicoscinium cf. discretum (Prantl, 1932). - Suárez Andrés, partim (non pl. 1, fig. 8, p. 191, non pl. 2, figs 1-4, p. 193).

2012 Fenestrapora occidentalis Ulrich, 1890. - Ernst, p. 229 , figs $15 \mathrm{f}-\mathrm{h}, 16 \mathrm{a}-\mathrm{e}$.

Material. - Nineteen specimens, DGO 12837-12855.

Description. - Exterior: Zoarium reticulate, conical, ob- verse on the outer surface. Branches sinuous, occasionally straight; dissepiments short and wide. Keel high, separating two row of autozooecia; apertures rounded, peristome incomplete. Fenestrules elliptical to oval, medium sized. Superstructure a terminal expansion of the keel, with scalloped flanks.

Interior: Autozooecial chambers rectangular, rhombic or polygonal, short and high, larger and rounded at dissepiment junctions. Axial wall straight or slightly zigzag. Hemisepta lacking. Basal plate concave, with few longitudinal reverse ridges, commonly one beneath the axial wall. Granular core of superstructure forming a sharp ridge on top of the keel; regularly spaced microstyles on both flanks. Laminar skeleton well developed, pierced by abundant micro- and macrostyles. Possible reproductive heterozooecia (brood chambers). Aviculomorphs (McKinney 1998) on the reverse of branches, obverse of dissepiments and possibly also occupying lateral positions in the superstructure.

Remarks. $-F$. occidentalis differs from $F$. transcaucasica Morozova \& Lavrentjeva, 1998 from the upper Eifelian of Transcaucasia, in having smaller fenestrules and shorter, narrower fenestrules.

Occurrence. - Middle Devonian (Hamilton), USA; Emsian-lower Eifelian, Arnao and Moniello Inlet, Cantabrian Zone, NW Spain.

Family Septoporidae Morozova, 1962

Genus Paraseptopora Lisitsyn, 1991

Type species. - Pinnatopora uralica Nikiforova, 1938; Lower Permian, Urals (URSS).

Paraseptopora geometrica sp. nov. Figure 7A-E, Table 11

1999 Septopora sp. A. - Suárez Andrés, pp. 186-187, pl. 1, figs 4-6, pl. 2, fig. 1.

Holotype. - DGO 12968.

Paratypes. - DGO 12969-12972.

Type horizon and locality. - Upper Emsian-lower Eifelian. Moniello Formation, Arnao.

Material. - Five specimens, DGO 12968-12972.

Etymology. - The name refers to the regular arrangement and growth of pinnae. Gender feminine. 
Table 11. Measurements of Paraseptopora geometrica sp. nov. Abbreviations as in Table 1 .

\begin{tabular}{|c|c|c|c|c|c|c|}
\hline & $\mathrm{X}$ & SD & $\mathrm{CV}$ & MAX & MIN & $\mathrm{N}$ \\
\hline Branch thickness (mm) & 0.70 & 0.082 & 0.0068 & 0.76 & 0.51 & 8 \\
\hline Branch width (mm) & 0.44 & 0.060 & 0.0036 & 0.54 & 0.32 & 10 \\
\hline $\begin{array}{l}\text { Distance between branch } \\
\text { centres }(\mathrm{mm})\end{array}$ & 1.03 & 0.354 & 0.1251 & 1.47 & 0.51 & 11 \\
\hline $\begin{array}{l}\text { Aperture spacing across } \\
\text { branch }(\mathrm{mm})\end{array}$ & 0.29 & 0.039 & 0.0015 & 0.37 & 0.21 & 27 \\
\hline $\begin{array}{l}\text { Aperture spacing along } \\
\text { branch }(\mathrm{mm})\end{array}$ & 0.29 & 0.027 & 0.0007 & 0.36 & 0.23 & 28 \\
\hline Aperture width (mm) & 0.12 & 0.012 & 0.0001 & 0.15 & 0.10 & 21 \\
\hline $\begin{array}{l}\text { Maximal chamber width } \\
(\mathrm{mm})\end{array}$ & 0.14 & 0.012 & 0.0001 & 0.16 & 0.12 & 8 \\
\hline Chamber depth (mm) & 0.18 & 0.009 & 0.0001 & 0.19 & 0.18 & 3 \\
\hline Chamber length (mm) & 0.25 & 0.019 & 0.0003 & 0.28 & 0.22 & 8 \\
\hline $\begin{array}{l}\text { Angle transverse } \\
\text { wall-basal plate } \\
\text { (degrees) }\end{array}$ & 63.69 & 1.48 & 2.19 & 64.73 & 62.64 & 2 \\
\hline Pinnae width, mm & 0.42 & 0.047 & 0.0022 & 0.51 & 0.36 & 15 \\
\hline $\begin{array}{l}\text { Distance between pinnae } \\
\text { centers }(\mathrm{mm})\end{array}$ & 0.97 & 0.125 & 0.0156 & 1.19 & 0.71 & 19 \\
\hline $\begin{array}{l}\text { Aperture spacing across } \\
\text { pinna }(\mathrm{mm})\end{array}$ & 0.28 & 0.048 & 0.0023 & 0.35 & 0.22 & 9 \\
\hline $\begin{array}{l}\text { Aperture spacing along } \\
\text { pinna }(\mathrm{mm})\end{array}$ & 0.27 & 0.018 & 0.0003 & 0.31 & 0.24 & 12 \\
\hline $\begin{array}{l}\text { Aperture width in pinnae } \\
(\mathrm{mm})\end{array}$ & 0.11 & 0.015 & 0.0002 & 0.14 & 0.09 & 9 \\
\hline $\begin{array}{l}\text { Maximal chamber width } \\
\text { in pinnae }(\mathrm{mm})\end{array}$ & 0.13 & 0.006 & 0.0000 & 0.13 & 0.12 & 3 \\
\hline $\begin{array}{l}\text { Chamber length in } \\
\text { pinnae (mm) }\end{array}$ & 0.23 & 0.019 & 0.0004 & 0.25 & 0.21 & 3 \\
\hline $\begin{array}{l}\text { Angle branch-pinna } \\
\text { (degrees) }\end{array}$ & 53.65 & 8.81 & 77.56 & 70.02 & 34.03 & 19 \\
\hline
\end{tabular}

Diagnosis. - Paraseptopora with straight main branches and short, straight, regularly arranged pinnae.

Description. - Exterior: Zoarium reticulate, fan-shaped. Main branches straight or widely curved; pinnae short and straight. Some pinnae change growth direction paralleling main growth direction and become main branches. Keel very low, nodes absent. Two rows of autozooecia per branch, apertures rounded, with peristome. Fenestrules moderately large, regular.

Interior: Autozooecial chambers subrectangular, sac-shaped in longitudinal section, vestibule wide, subvertical. Axial wall straight. Hemisepta lacking. Basal plate concave, with few reverse ridges. Granular skeleton thin and short in the keel; microstyles abundant. Heterozooecia not observed.

Remarks. - P. geometrica sp. nov. differs from P. irregularis sp. nov. in the regular arrangement of the meshwork,
Table 12. Measurements of Paraseptopora irregularis sp. nov. Abbreviations as in Table 1.

\begin{tabular}{|c|c|c|c|c|c|c|}
\hline & $\mathrm{X}$ & SD & $\mathrm{CV}$ & MAX & MIN & $\mathrm{N}$ \\
\hline Branch thickness (mm) & 0.69 & 0.081 & 0.0066 & 0.78 & 0.56 & 5 \\
\hline Branch width (mm) & 0.51 & 0.065 & 0.0043 & 0.60 & 0.41 & 13 \\
\hline $\begin{array}{l}\text { Distance between branch } \\
\text { centres }(\mathrm{mm})\end{array}$ & 1.32 & 0.310 & 0.0958 & 1.85 & 0.87 & 22 \\
\hline $\begin{array}{l}\text { Aperture spacing across } \\
\text { branch }(\mathrm{mm})\end{array}$ & 0.29 & 0.040 & 0.0016 & 0.35 & 0.18 & 28 \\
\hline $\begin{array}{l}\text { Aperture spacing along } \\
\text { branch }(\mathrm{mm})\end{array}$ & 0.30 & 0.038 & 0.0014 & 0.37 & 0.22 & 28 \\
\hline Aperture width (mm) & 0.12 & 0.011 & 0.0001 & 0.14 & 0.10 & 26 \\
\hline $\begin{array}{l}\text { Maximal chamber width } \\
(\mathrm{mm})\end{array}$ & 0.13 & 0.012 & 0.0001 & 0.15 & 0.11 & 20 \\
\hline Chamber length (mm) & 0.28 & 0.024 & 0.0006 & 0.31 & 0.23 & 20 \\
\hline $\begin{array}{l}\text { Angle transverse } \\
\text { wall-basal plate } \\
\text { (degrees) }\end{array}$ & 58.62 & 3.89 & 15.16 & 61.37 & 55.86 & 2 \\
\hline Pinnae width (mm) & 0.41 & 0.078 & 0.0061 & 0.58 & 0.30 & 14 \\
\hline $\begin{array}{l}\text { Distance between pinnae } \\
\text { centres }(\mathrm{mm})\end{array}$ & 1.03 & 0.155 & 0.0239 & 1.28 & 0.69 & 23 \\
\hline $\begin{array}{l}\text { Aperture spacing across } \\
\text { pinna }(\mathrm{mm})\end{array}$ & 0.28 & 0.019 & 0.0004 & 0.30 & 0.26 & 3 \\
\hline $\begin{array}{l}\text { Aperture spacing along } \\
\text { pinna }(\mathrm{mm})\end{array}$ & 0.32 & 0.011 & 0.0001 & 0.33 & 0.31 & 2 \\
\hline $\begin{array}{l}\text { Maximal chamber width } \\
\text { in pinnae }(\mathrm{mm})\end{array}$ & 0.15 & 0.054 & 0.0030 & 0.27 & 0.12 & 7 \\
\hline $\begin{array}{l}\text { Chamber length in } \\
\text { pinnae (mm) }\end{array}$ & 0.25 & 0.056 & 0.0031 & 0.31 & 0.15 & 7 \\
\hline $\begin{array}{l}\text { Angle branch-pinna } \\
\text { (degrees) }\end{array}$ & 53.09 & 7.47 & 55.72 & 67.41 & 34.95 & 31 \\
\hline
\end{tabular}

straight pinnae, and slightly narrower main branches with a shorter spacing. $P$. geometrica is similar to $P$. variabilis (Nekhoroshev, 1977) in the absence of nodes, but differs in having wider branches and pinnae, somewhat larger fenestrules and larger spacing of zooidal apertures.

Occurrence. - Upper Emsian-lower Eifelian, Arnao, Cantabrian Zone, NW Spain.

Paraseptopora irregularis sp. nov.

Figure 7F-J, Table 12

Holotype. - DGO 12973.

Paratypes. - DGO 12975, 12977, 12978.

Type horizon and locality. - Upper Emsian-lower Eifelian, Moniello Formation, Arnao.

Material. - Seven specimens, DGO 12973-12979.

Etymology. - From Latin, referring to the irregular arrangement of pinnae. Gender feminine. 
Table 13. Measurements of Penniretepora bohemica (Prantl, 1932). Abbreviations as in Table 1.

\begin{tabular}{|c|c|c|c|c|c|c|}
\hline & $\mathrm{X}$ & SD & $\mathrm{CV}$ & MAX & MIN & $\mathrm{N}$ \\
\hline Branch thickness (mm) & 1.42 & 0.443 & 0.1964 & 2.40 & 0.96 & 8 \\
\hline Branch width (mm) & 0.94 & 0.461 & 0.2126 & 2.21 & 0.52 & 12 \\
\hline $\begin{array}{l}\text { Aperture spacing across } \\
\text { branch }(\mathrm{mm})\end{array}$ & 0.33 & 0.054 & 0.0030 & 0.46 & 0.23 & 64 \\
\hline $\begin{array}{l}\text { Aperture spacing along } \\
\text { branch }(\mathrm{mm})\end{array}$ & 0.28 & 0.033 & 0.0011 & 0.35 & 0.21 & 57 \\
\hline Aperture width (mm) & 0.11 & 0.015 & 0.0002 & 0.14 & 0.08 & 50 \\
\hline $\begin{array}{l}\text { Maximal chamber width } \\
(\mathrm{mm})\end{array}$ & 0.14 & 0.013 & 0.0002 & 0.16 & 0.11 & 42 \\
\hline Chamber depth (mm) & 0.13 & 0.025 & 0.0006 & 0.19 & 0.10 & 10 \\
\hline Chamber length (mm) & 0.23 & 0.032 & 0.0011 & 0.30 & 0.17 & 42 \\
\hline $\begin{array}{l}\text { Angle transverse } \\
\text { wall-basal plate } \\
\text { (degrees) }\end{array}$ & 74.22 & 3.62 & 13.07 & 80.70 & 69.77 & 7 \\
\hline Keel node spacing (mm) & 0.95 & 0.230 & 0.0531 & 1.29 & 0.61 & 7 \\
\hline Pinnae width (mm) & 0.43 & 0.062 & 0.0039 & 0.53 & 0.34 & 8 \\
\hline $\begin{array}{l}\text { Distance between pinnae } \\
\text { centres }(\mathrm{mm})\end{array}$ & 0.78 & 0.113 & 0.0128 & 0.97 & 0.56 & 24 \\
\hline $\begin{array}{l}\text { Aperture spacing across } \\
\text { pinna }(\mathrm{mm})\end{array}$ & 0.27 & 0.036 & 0.0013 & 0.35 & 0.21 & 15 \\
\hline $\begin{array}{l}\text { Aperture spacing along } \\
\text { pinna }(\mathrm{mm})\end{array}$ & 0.29 & 0.086 & 0.0073 & 0.53 & 0.22 & 17 \\
\hline $\begin{array}{l}\text { Maximal chamber width } \\
\text { in pinnae }(\mathrm{mm})\end{array}$ & 0.14 & 0.035 & 0.0012 & 0.26 & 0.11 & 38 \\
\hline $\begin{array}{l}\text { Chamber length in } \\
\text { pinnae }(\mathrm{mm})\end{array}$ & 0.19 & 0.025 & 0.0006 & 0.25 & 0.12 & 38 \\
\hline $\begin{array}{l}\text { Angle branch-pinna } \\
\text { (degrees) }\end{array}$ & 61.77 & 5.55 & 30.83 & 70.79 & 51.28 & 31 \\
\hline
\end{tabular}

Diagnosis. - Paraseptopora with sinuous main branches and curved pinnae producing irregular or heart-shaped fenestrules.

Description. - Ex terior: Zoarium reticulate, fan-shaped; main branches straight or widely arched. Pinnae short, curved, irregular in length; some become main branches and develop pinnae. Keel very low, without nodes, separating two rows of autozooecia; zooecial apertures circular, with peristome. Fenestrules large, irregular in shape.

Interior: Autozooecial chambers rounded rectangularpentagonal, sac-shaped in longitudinal section, vestibule wide and tilted towards fenestrule. Axial wall straight or slightly sinuous. Hemisepta absent. Basal plate concave, with 2-3 prominent reverse ridges. Microstyles thin, macrostyles absent. Heterozooecia not observed.

Remarks. - P. irregularis sp. nov. differs from P. geometrica sp. nov. in having larger, irregular or heartshaped fenestrules, curved pinnae and a larger branch spacing.

Occurrence. - Emsian-lower Eifelian, Arnao and Moniello Inlet, Cantabrian Zone, NW Spain.

Family Acanthocladiidae Zittel, 1880

\section{Genus Penniretepora d'Orbigny, 1849}

Type species. - Retepora pluma Phillips, 1836; Visean, Yorkshire, England and Ireland.

\section{Penniretepora bohemica (Prantl, 1932)}

Figure 8A-D, Table 13

1932 Glauconome bohemica; Prantl, pp. 29-30, pl. 3, figs $15-16$.

1986 Penniretepora bohemica (Prantl, 1932). - McKinney \& Kř́ž, p. 78, fig. 48.

1999 Penniretepora sp. A. - Suárez Andrés \& González Álvarez, p. 646, pl. 1, figs 8-10.

Description. - Exterior: Zoarium pinnate, main branch straight or arched; pinnae short and straight. Some pinnae may become secondary main branches. Keel low with a single row of regularly spaced nodes, separating two rows of autozooecia. Zooecial apertures rounded, with a complete peristome.

Interior: Autozooecial chambers pentagonal or barrelshaped, low, with a long vestibule. Proximal hemiseptum weak. Axial wall zigzag, transverse walls well developed. Basal plate concave with several small reverse ridges. Microstyles numerous, macrostyles absent on the reverse. Strong thickening of laminar skeleton in main branches. Heterozooecia not observed.

Remarks. - The studied material is assigned to the species $P$. bohemica on account of the arrangement of pinnae,

Figure 7. A-E-Paraseptopora geometrica sp. nov., Moniello Formation. • A - obverse surface of a colony, DGO 12971 . B - shallow tangential section, holotype, DGO 12968. • C - longitudinal section of a main branch, DGO 12970 . $\bullet$ D - transverse section of a colony cutting four main branches, holotype, DGO 12968. E - tangential section showing autozooecial morphology, DGO 12968 . $\bullet$ F-J - Paraseptopora irregularis sp. nov. Moniello Formation. $\bullet \mathrm{F}$ - transverse section cutting main branches and pinnae, DGO 12975 . $\bullet \mathrm{G}-\mathrm{J}$ - holotype, DGO 12973 ; G - tangential section showing the irregular meshwork; $\mathrm{H}$ - tangential section; chamber morphology and curved pinnae can be observed; I-shallow tangential section; $\mathrm{J}$ - longitudinal section of a main branch. 
Juan Luis Suárez Andrés \& Patrick N. Wyse Jackson • Lower-Middle Devonian fenestrate Bryozoa (NW Spain)
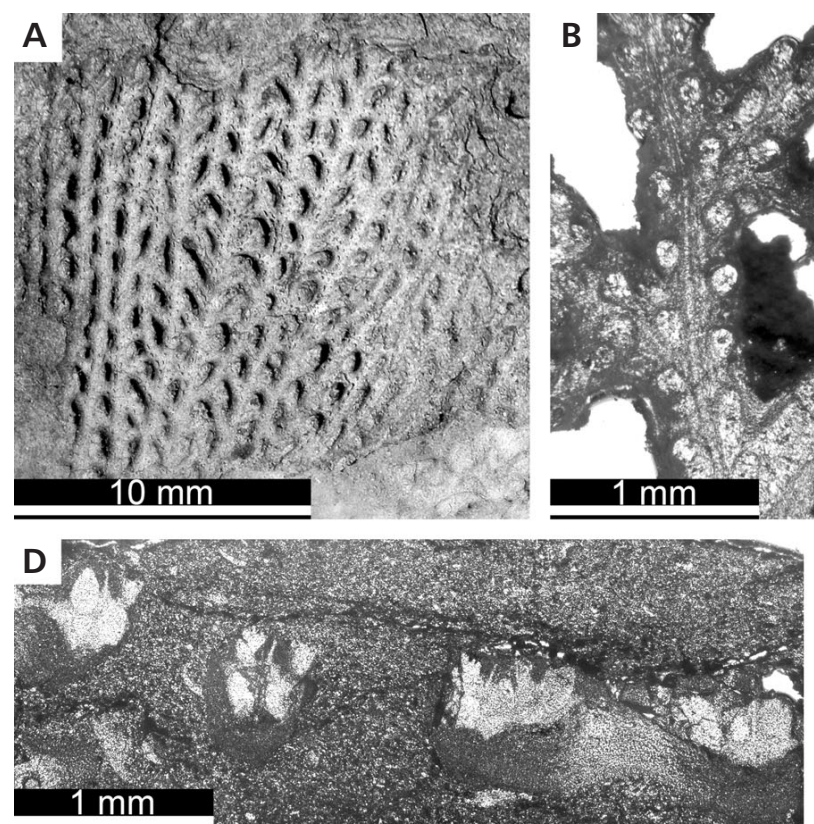

E
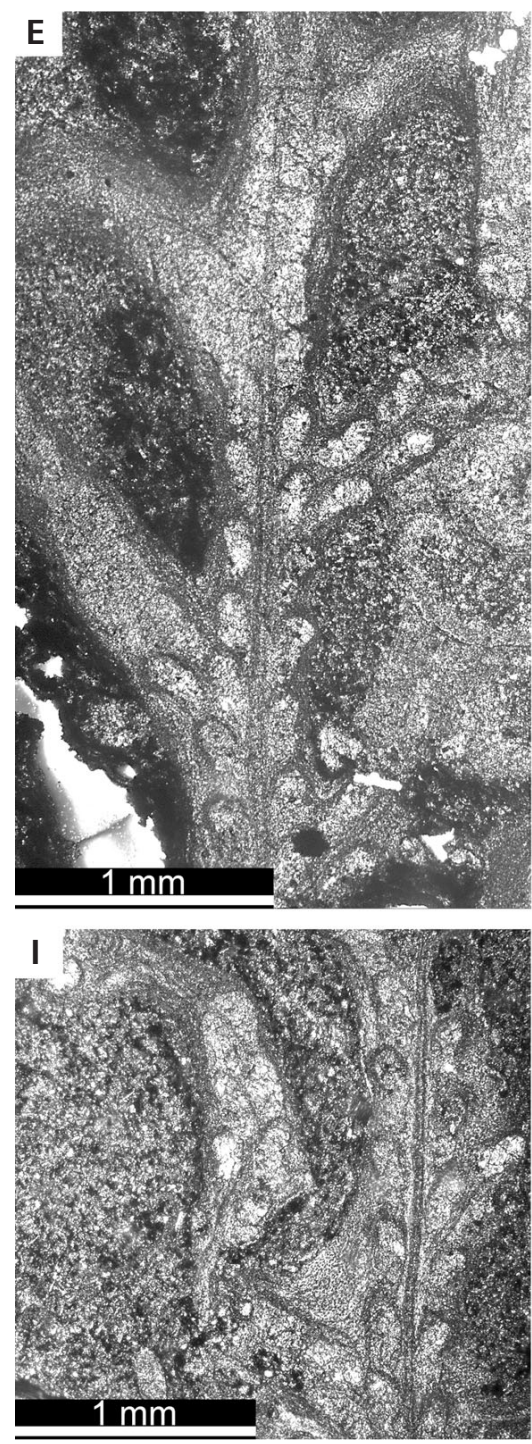
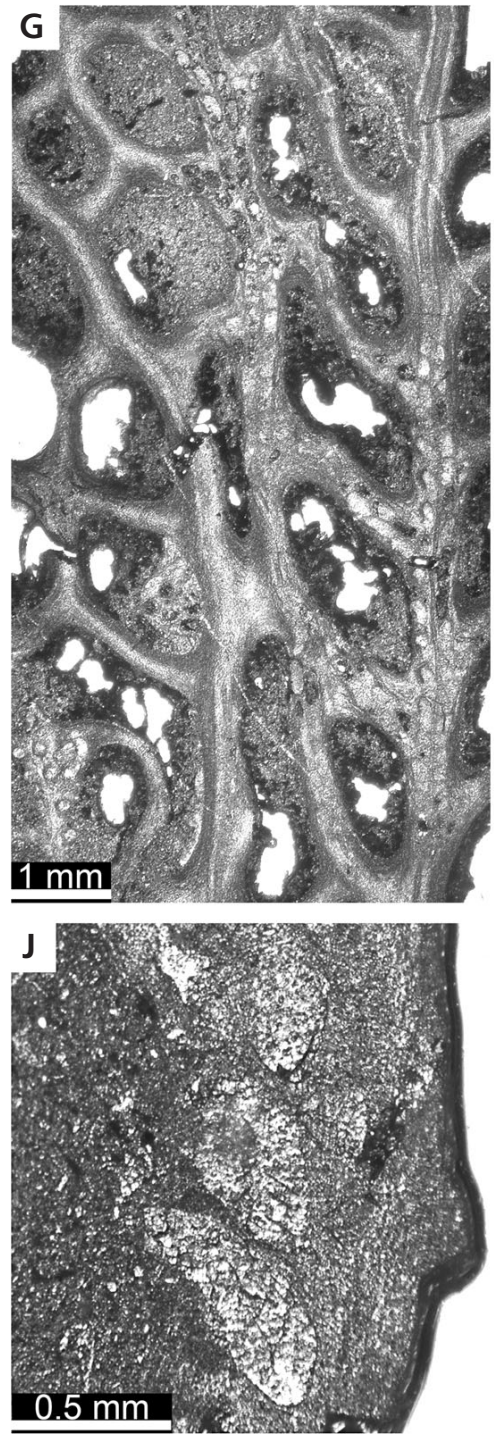

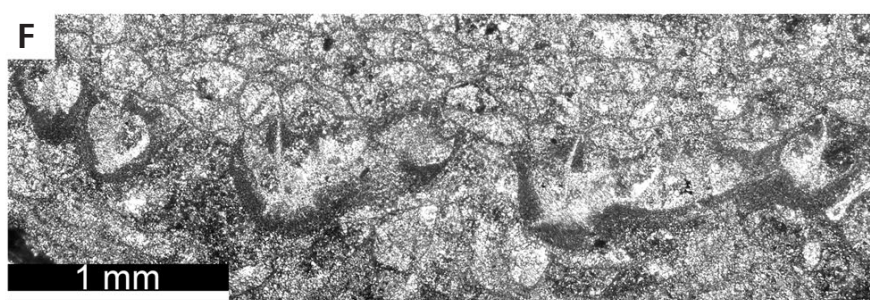

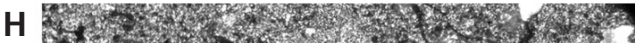

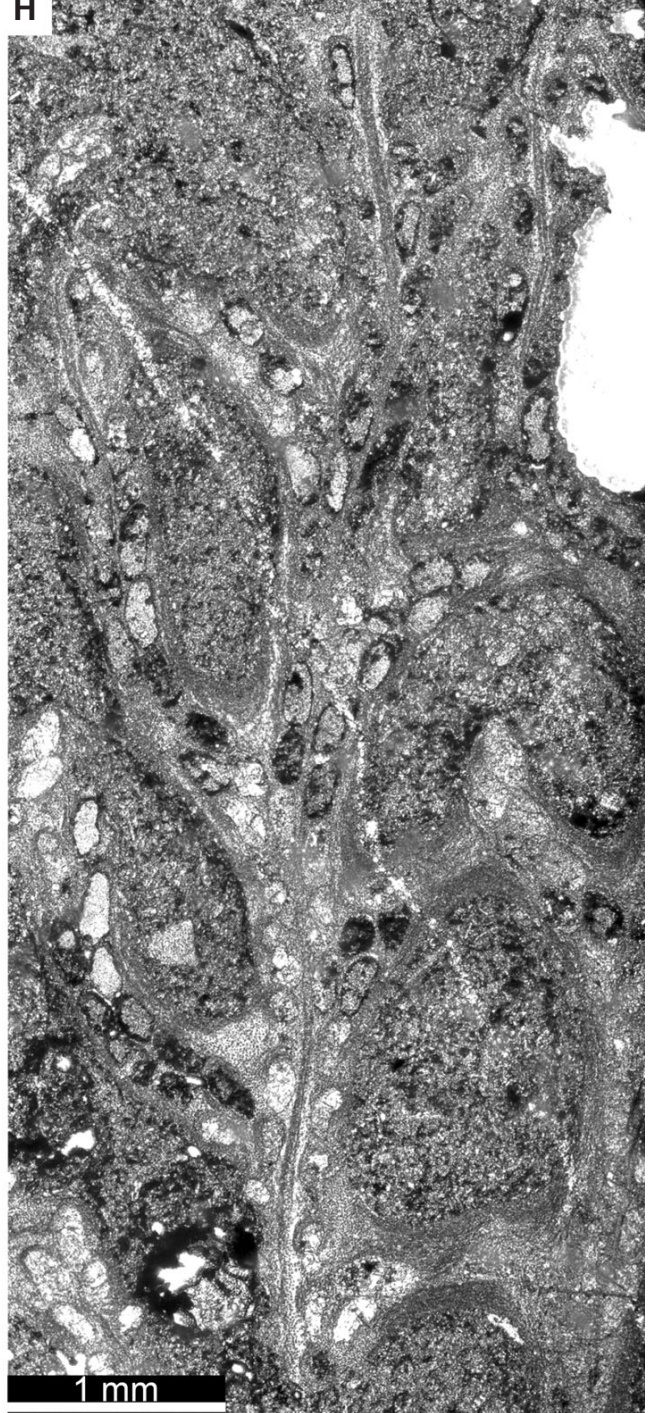


Table 14. Measurements of Kalvariella antiqua sp. nov. Abbreviations as in Table 1 .

\begin{tabular}{|c|c|c|c|c|c|c|}
\hline & $\mathrm{X}$ & SD & $\mathrm{CV}$ & MAX & MIN & $\mathrm{N}$ \\
\hline Branch thickness (mm) & 0.82 & 0.207 & 0.0429 & 0.97 & 0.67 & 2 \\
\hline Branch width (mm) & 0.60 & 0.008 & 0.0001 & 0.60 & 0.59 & 3 \\
\hline $\begin{array}{l}\text { Aperture spacing across } \\
\text { branch }(\mathrm{mm})\end{array}$ & 0.34 & 0.021 & 0.0004 & 0.38 & 0.30 & 23 \\
\hline $\begin{array}{l}\text { Aperture spacing along } \\
\text { branch }(\mathrm{mm})\end{array}$ & 0.33 & 0.019 & 0.0004 & 0.37 & 0.30 & 22 \\
\hline Aperture width (mm) & 0.10 & 0.009 & 0.0001 & 0.12 & 0.087 & 24 \\
\hline $\begin{array}{l}\text { Maximal chamber width } \\
(\mathrm{mm})\end{array}$ & 0.15 & 0.011 & 0.0001 & 0.17 & 0.13 & 11 \\
\hline Chamber length (mm) & 0.29 & 0.019 & 0.0004 & 0.32 & 0.25 & 11 \\
\hline Pinnae width (mm) & 0.57 & 0.039 & 0.0015 & 0.62 & 0.53 & 4 \\
\hline $\begin{array}{l}\text { Distance between pinnae } \\
\text { centres }(\mathrm{mm})\end{array}$ & 0.82 & 0.108 & 0.0117 & 0.94 & 0.61 & 7 \\
\hline $\begin{array}{l}\text { Aperture spacing across } \\
\text { pinna }(\mathrm{mm})\end{array}$ & 0.18 & 0.021 & 0.0004 & 0.21 & 0.14 & 11 \\
\hline $\begin{array}{l}\text { Aperture spacing along } \\
\text { pinna }(\mathrm{mm})\end{array}$ & 0.19 & 0.020 & 0.0004 & 0.21 & 0.16 & 6 \\
\hline $\begin{array}{l}\text { Maximal chamber width } \\
\text { in pinnae }(\mathrm{mm})\end{array}$ & 0.15 & 0.008 & 0.0001 & 0.15 & 0.14 & 3 \\
\hline $\begin{array}{l}\text { Chamber length in } \\
\text { pinnae }(\mathrm{mm})\end{array}$ & 0.30 & 0.051 & 0.0026 & 0.35 & 0.25 & 3 \\
\hline $\begin{array}{l}\text { Angle branch-pinna } \\
\text { (degrees) }\end{array}$ & 58.60 & 4.78 & 22.80 & 65.26 & 50.86 & 6 \\
\hline
\end{tabular}

distance between apertures, and chamber shape and width. $P$. bohemica differs from $P$. spinosa (Počta, 1894) from the Lower Devonian of Prague, in the shape and dimensions of autozooecial chambers, as well as in having wider pinnae (McKinney \& Kř́̌̌ 1986).

Occurrence. - Emsian, Prague Basin, Czech Republic. Emsian-lower Eifelian, Arnao, Cantabrian Zone, NW Spain.

\section{Genus Kalvariella Morozova, 1970}

Type species. - Kalvariella typica Morozova, 1970; Upper Permian, Lithuania.

Kalvariella antiqua sp. nov. Figure 8E-H, Table 14

Holotype. - DGO 12871.
Paratypes. - DGO 12989-12991.

Type horizon and locality. - Upper Emsian-lower Eifelian, Moniello Formation, Arnao.

Material. - Five specimens, DGO 12871, 12988-12991.

Etymology. - From Latin antiqua, ancient. Gender feminine.

Diagnosis. - Kalvariella forming small zoaria with relatively thin biserial main branch and very short multiserial pinnae.

Description. - Exterior: Zoarium pinnate, main branch relatively thin, with very short opposite or alternating pinnae. Keel low in main branch, with a single row of nodes; keel absent in pinnae. Two rows of autozooecia in main branch, 3-4 in pinnae. Zooecial apertures stellate, peristome well defined.

Interior: Autozooecial chambers rectangular or parallelogram-shaped in main branch, locally pentagonal; axial wall straight, locally zigzag. Chambers elongate hexagonal or rhombic in pinnae, irregular in the first generation close to main branch. Hemisepta absent. Basal plate concave, very short and curved towards the reverse in pinnae, bearing few generations of autozooecia. Microstyles common, thin. Laminar skeleton thickened in main branch. Heterozooecia not observed.

Remarks. - K. antiqua sp. nov. can be distinguished from the pinnate fauna among which it occurs in the presence of multiserial pinnae. From the five known species of Kalvariella it differs in having very short, backward curved pinnae. One of the specimens corresponds to the basal part of a mature colony with astogenetic thickening of laminar skeleton and shows bioclaustration structures identified as Caupokeras caused by growth of a modular symbiont organism on the obverse surface of the bryozoan colony. This is the only reported case of Caupokeras developed on a superstructure-lacking fenestrate (Suárez Andrés 2014).

Occurrence. - Emsian-lower Eifelian, Arnao and Moniello Inlet, Cantabrian Zone, NW Spain.

Figure 8. A-D - Penniretepora bohemica (Prantl, 1932), Moniello Formation. • A-C - DGO 12980; A - transverse section of a heavily thickened main branch; B - shallow tangential section showing zooecial apertures and keel nodes (arrows); C - longitudinal section of a main branch. $\bullet$ D - deep tangential section cutting through zooecial chambers and basal plate, DGO 12985 . $\bullet$ E-H - Kalvariella antiqua sp. nov., Moniello Formation. $\bullet$ E - deep tangential section showing short multiserial pinnae, DGO 12990. • F - obverse surface of a secondary main branch with multiseral pinnae, holotype, DGO 12871. $\bullet \mathrm{G}$ - deep tangential section, DGO 12990 . $\bullet \mathrm{H}$-transverse section cutting a main branch; the basal plate of pinnae is curved towards the reverse, DGO 12990. 
Juan Luis Suárez Andrés \& Patrick N. Wyse Jackson • Lower-Middle Devonian fenestrate Bryozoa (NW Spain)

A

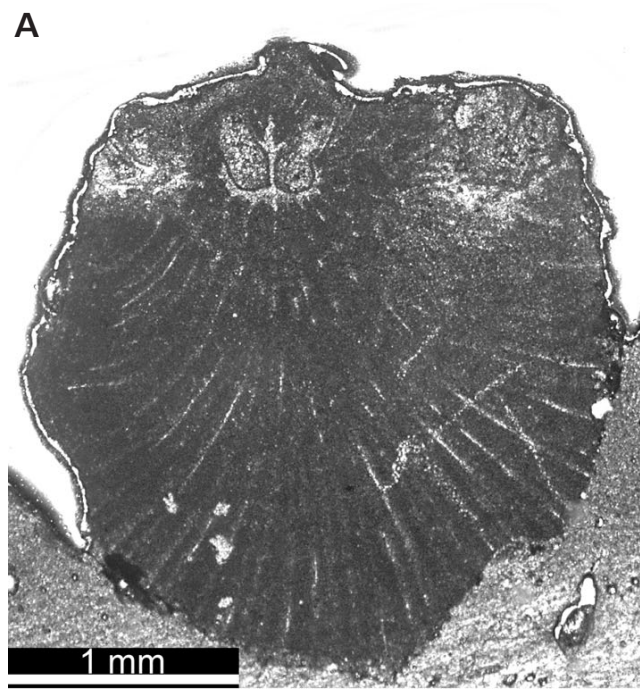

D X X

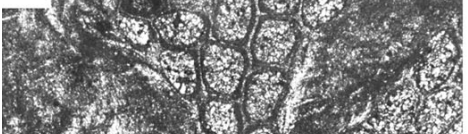

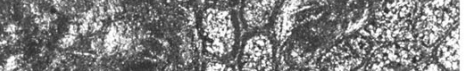

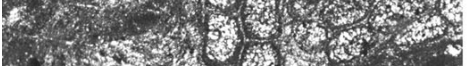
- $3 x+2$ \%. $x=2$ $-5,3)$ Wh

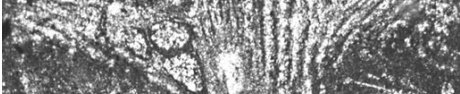

12.2. (6. w)

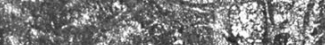

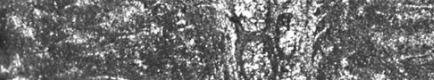

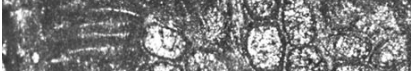

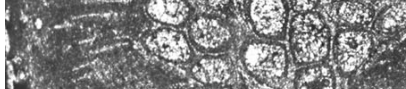

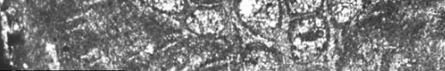
$\left.0.5 \mathrm{~mm}-3 \mathrm{~m}^{2} \mathrm{r}\right)$

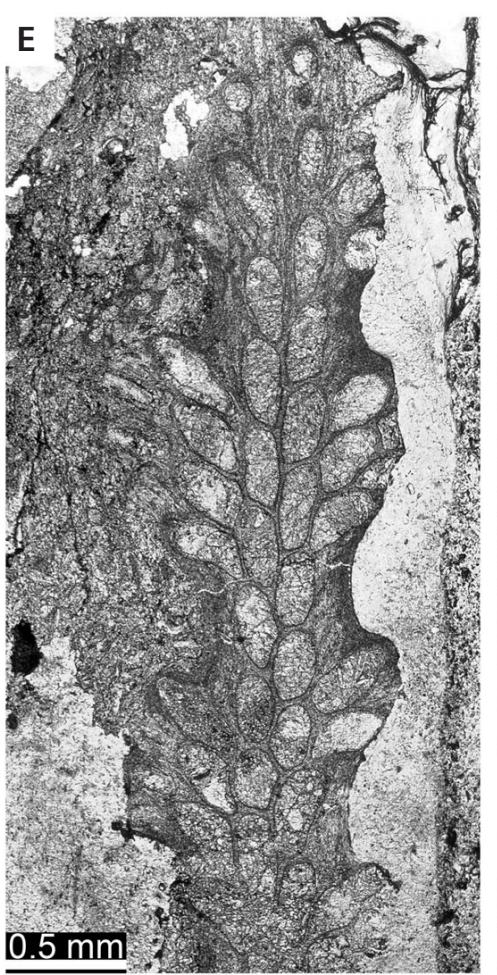

$1 \mathrm{~mm}$
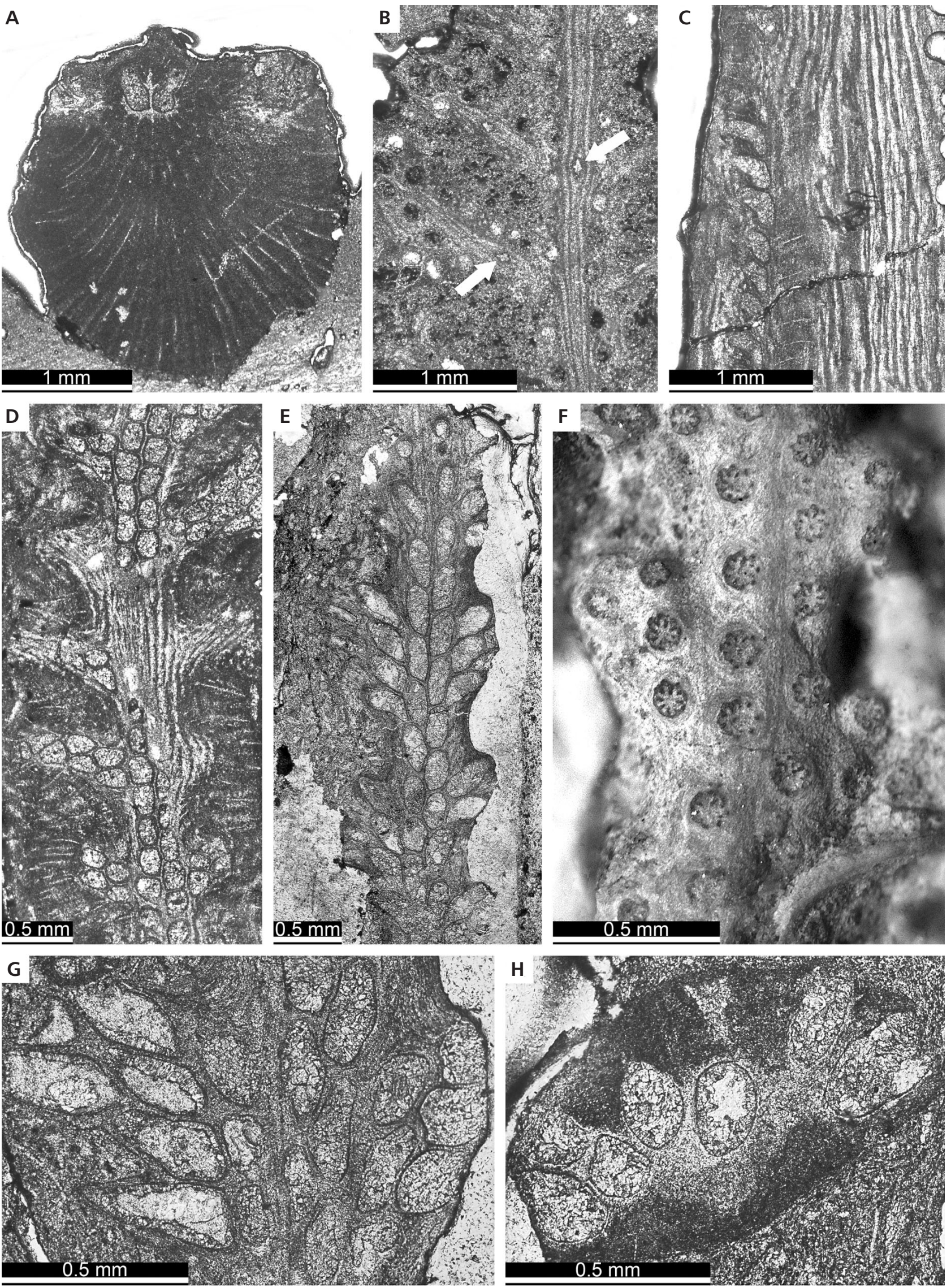
Table 15. Measurements of Filites robustus sp. nov. Abbreviations as in Table 1.

\begin{tabular}{|c|c|c|c|c|c|c|}
\hline & $\mathrm{X}$ & SD & $\mathrm{CV}$ & MAX & MIN & $\mathrm{N}$ \\
\hline Branch thickness (mm) & 0.85 & 0.107 & 0.0115 & 0.99 & 0.67 & 9 \\
\hline Branch width (mm) & 0.73 & 0.129 & 0.0166 & 0.96 & 0.56 & 34 \\
\hline $\begin{array}{l}\text { Aperture spacing across } \\
\text { branch }(\mathrm{mm})\end{array}$ & 0.27 & 0.031 & 0.0010 & 0.34 & 0.21 & 43 \\
\hline $\begin{array}{l}\text { Aperture spacing along } \\
\text { branch }(\mathrm{mm})\end{array}$ & 0.40 & 0.053 & 0.0028 & 0.55 & 0.29 & 47 \\
\hline Aperture width (mm) & 0.13 & 0.017 & 0.0003 & 0.16 & 0.08 & 56 \\
\hline $\begin{array}{l}\text { Maximal chamber width } \\
(\mathrm{mm})\end{array}$ & 0.15 & 0.020 & 0.0004 & 0.20 & 0.11 & 93 \\
\hline Chamber depth (mm) & 0.23 & 0.011 & 0.0001 & 0.24 & 0.23 & 2 \\
\hline Chamber length (mm) & 0.28 & 0.037 & 0.0014 & 0.39 & 0.21 & 93 \\
\hline $\begin{array}{l}\text { Angle transverse } \\
\text { wall-basal plate } \\
\text { (degrees) }\end{array}$ & 74.77 & 3.96 & 15.64 & 77.57 & 71.98 & 2 \\
\hline Pinnae width (mm) & 0.54 & 0.098 & 0.0096 & 0.79 & 0.43 & 22 \\
\hline $\begin{array}{l}\text { Aperture spacing across } \\
\text { pinna }(\mathrm{mm})\end{array}$ & 0.84 & 0.086 & 0.0073 & 1.09 & 0.67 & 46 \\
\hline $\begin{array}{l}\text { Aperture spacing along } \\
\text { pinna }(\mathrm{mm})\end{array}$ & 0.23 & 0.009 & 0.0001 & 0.24 & 0.23 & 2 \\
\hline $\begin{array}{l}\text { Maximal chamber width } \\
\text { in pinnae }(\mathrm{mm})\end{array}$ & 0.24 & 0.044 & 0.0020 & 0.34 & 0.15 & 55 \\
\hline $\begin{array}{l}\text { Chamber length in } \\
\text { pinnae }(\mathrm{mm})\end{array}$ & 0.13 & 0.025 & 0.0006 & 0.18 & 0.09 & 55 \\
\hline $\begin{array}{l}\text { Chamber depth in } \\
\text { pinnae }(\mathrm{mm})\end{array}$ & 0.33 & 0.043 & 0.0019 & 0.40 & 0.24 & 22 \\
\hline $\begin{array}{l}\text { Angle branch-pinna } \\
\text { (degrees) }\end{array}$ & 71.69 & 6.48 & 42.00 & 81.41 & 56.91 & 30 \\
\hline
\end{tabular}

\section{Genus Filites Barrande, 1894 in Počta (1894)}

Type species. - Filites bohemicus Barrande, 1894 in Počta (1894); Early Devonian, Czech Republic.

Remarks. - One of the most evident characters of Filites bohemicus is the unusual backward recurved morphology of pinnae, which was included by McKinney \& Kříž (1986) in the diagnosis of the genus. Morozova (2001) emended these authors' diagnosis and assigned to Filites species previously attributed to Penniretepora with triangular autozooecial chambers, none of which showed vestigial, backward recurved pinnae. This is rejected here, and the specific composition of Filites is restricted to taxa in which zooecial chambers in pinnae exhibit a very different shape to that of chambers in main branch, as in the type species Filites bohemicus. This generic diagnostic characteristic can also be observed in the species Filites robustus sp. nov. defined herein.

Filites robustus sp. nov.

Figure 9A-E, Table 15

1999 Filites sp. A. - Suárez Andrés, pp. 190-192, pl. 2, figs $6-10$.

Holotype. - DGO 12941.

Paratypes. - DGO 12942-12950.

Type horizon and locality. - Upper Emsian-lower Eifelian, Moniello Formation, Arnao.

Material. - Fifteen specimens, DGO 12941-12955.

Etymology. - From Latin robustus, robust. Gender masculinum.

Diagnosis. - Filites with wide main branch, pinnae short and recurved with zooecia morphologically differentiated from those of main branch.

Description. - Exterior: Zoarium pinnate, pinnae short and curved backward, alternating on both flanks of main branch. Pinnae vestigial, short, curved backwards, rapidly tapering distally and merged with the reverse of main branch. Pinnae seldom grow straight and become secondary main branches approximately transversal to the parental one. Keel absent or very low and blunt. Two rows of autozooecia in main branches and pinnae; zooecial apertures rounded, with thin, very low peristome.

Interior: Autozooecial chambers dimorphic according to their placement in main branches or pinnae. Chambers triangular, triangular-pentagonal or trapezoidal, high and with a well developed vestibule in main branch. Chambers tubular, high, vesicular in tangential section, with a poorly differentiated vestibule. Hemisepta absent. Basal plate thin and concave; granular skeleton of axial wall not prolonged towards obverse. Microstyles very abundant, macrostyles absent. Laminar skeleton thickened, especially in main branch. Heterozooecia not observed.

Figure 9. A-E-Filites robustus sp. nov., Moniello Formation. • A - oblique tangential section, DGO 12951. • B - deep tangential section, DGO 12953. - $\mathrm{C}$ - longitudinal section of a colony cutting through three pinnae; autozooecial morphology progressively changed in the central pinna as it became a secondary main branch (arrow), DGO 12952 . $・$ D - transverse section cutting autozooecia in the main branch and in a pinna (left), DGO 12951 . E - longitudinal section, DGO 12953. • F-H - Polypora inusitata McKinney \& Křǐž, 1986, Moniello Formation. • F - transverse section of a branch prior to bifurcation, DGO 12999. Arnao. $\bullet \mathrm{G}$ - longitudinal section of a branch, DGO 13000. $\bullet$ H - tangential section, DGO 12995. 
Juan Luis Suárez Andrés \& Patrick N. Wyse Jackson • Lower-Middle Devonian fenestrate Bryozoa (NW Spain)
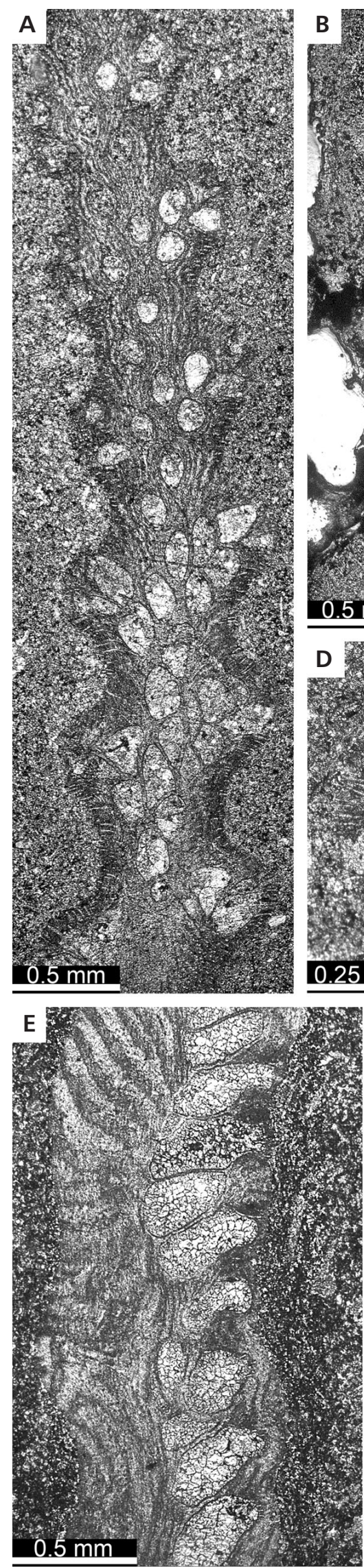
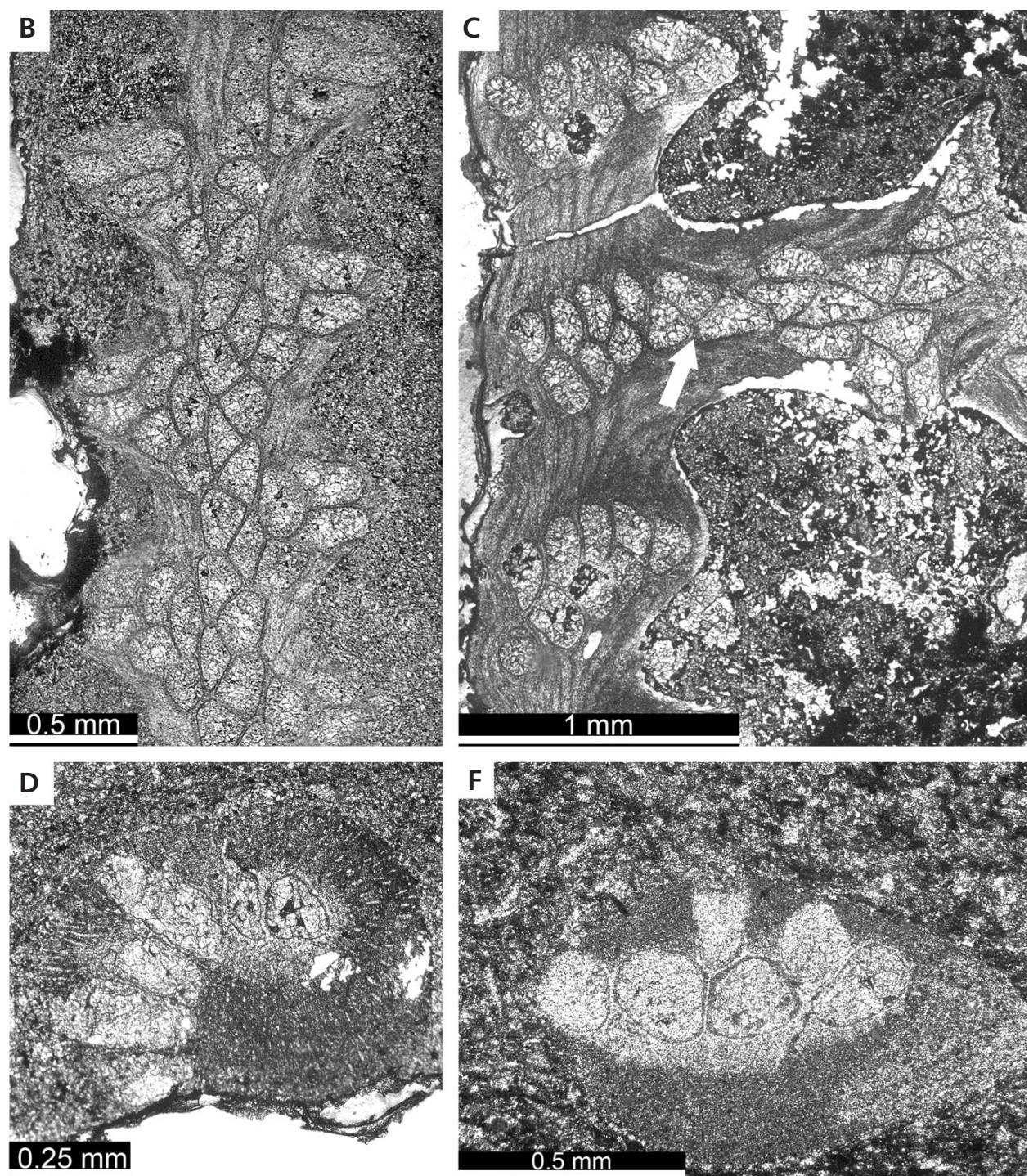
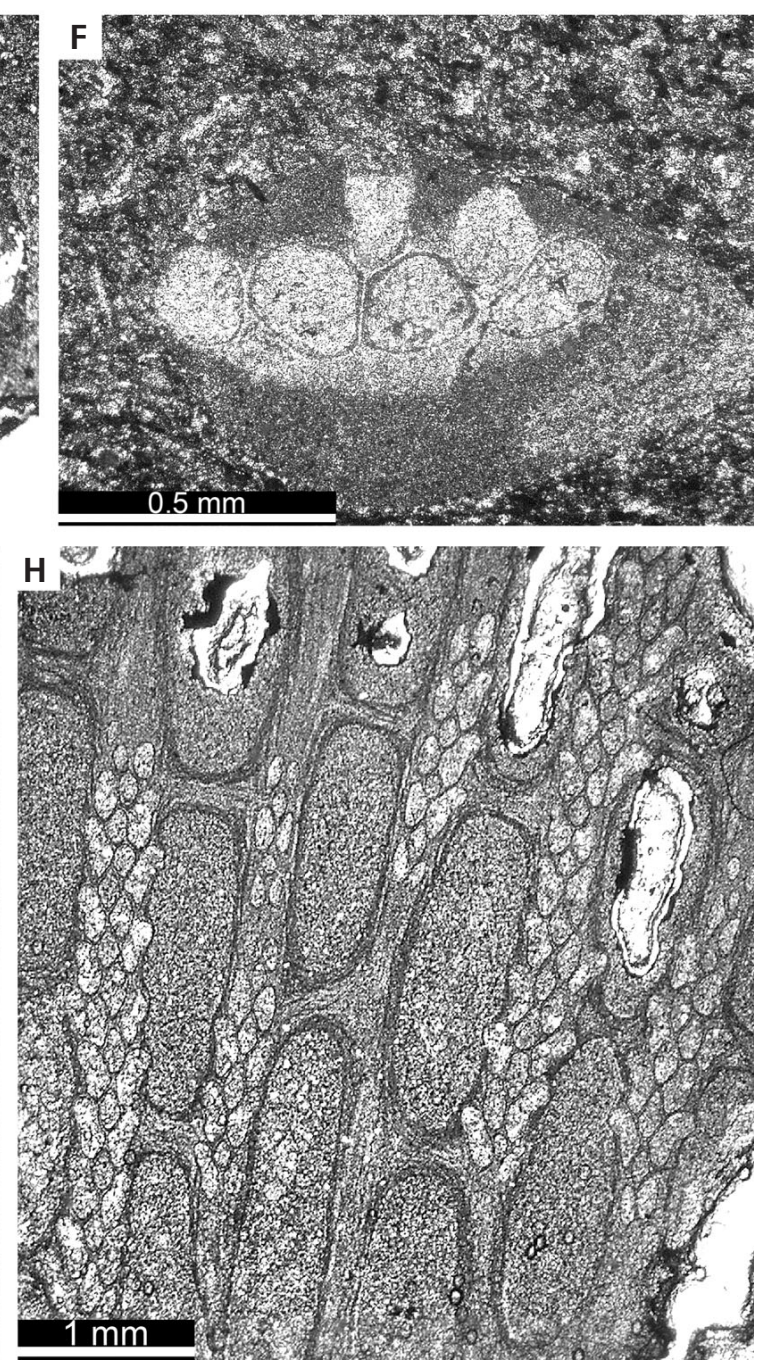
Remarks. $-F$. robustus sp. nov. differs from the type species, F. bohemicus, in having colonies with wider branches and pinnae, larger chambers and a greater spacing between zooecial apertures. Both species share the retrograde morphology of pinnae; Ernst \& Winkler Prins (2008) described and figured an undetermined fenestrate from the Carboniferous of the Cantabrian Zone (p. 46-47, pl. 29, figs 2-3) with a very similar external morphology, but unfortunately information about its internal organization is not available.

The growth pattern of $F$. robustus sp. nov. produced delicate colonies with main branches a few $\mathrm{cm}$ long, from which occasionally secondary main branches occur. The total lack of regularity in this aspect of colony growth contrasts with the internal organization of pinnae that become secondary main branches: the tubular chambers characteristic of pinnae gradually turned into the main branch type before the first secondary pinnae were formed.

Occurrence. - Emsian-lower Eifelian, Arnao and Moniello Inlet, Cantabrian Zone, NW Spain.

Family Polyporidae Vine, 1880

\section{Genus Polypora M'Coy, 1844}

Type species. - Polypora dendroides M'Coy, 1844; Tournaisian, Ireland.

\section{Polypora inusitata McKinney \& Kříž, 1986}

Figure 9F-H, Table 16

1986 Polypora inusitata; McKinney \& Kříž, 75-77, fig. 46a-f.

1999 Polypora aff. inusitata McKinney \& Kříž, 1986. Suárez Andrés, 187, pl. 1, figs 4-6, pl. 2, fig. 1.

Material. - Fourteen specimens, DGO 12992-13005.

Description. - Exterior: Zoarium reticulate, fan-shaped. Branches straight or slightly sinuous, dissepiments thin; 3-4 rows of zooecia per branch, zooecial apertures lobulated, arranged in rhombic pattern, peristome complete. Fenestrules moderately wide, very long.

Interior: Autozooecial chambers tubular, elongate rhomb- or hexagon-shaped in deep tangential section, proximal region recumbent. Hemisepta and diaphragms not observed. Basal plate concave, thin, with numerous small, thin reverse ridges. Laminar skeleton well developed, thickened on the reverse of branches, mainly along the axial plane. Microstyles abundant, macrostyles absent. Heterozooecia not observed.
Remarks. - The studied material resembles $P$. inusitata McKinney \& Křiž, 1986 in branch width and fenestrule length. The rectangular geometry of fenestrules in the Asturian colonies cannot be observed in the specimens figured by McKinney \& Kř́iž (1986). Nevertheless, the spacing of apertures is very similar, as well as chamber dimensions, for which reason the Asturian specimens have been assigned to this species. $P$. inusitata is similar to $P$. una Morozova, 1960 from the Givetian of the Kuznets Basin, but branches and zooecial apertures are more widely spaced.

Occurrence. - Emsian-lower Eifelian, Prague Basin, Czech Republic; Arnao and Moniello Inlet, Cantabrian Zone, NW Spain.

\section{Genus Anastomopora Simpson, 1897}

Type species. - Fenestella cinctuta Hall, 1884; Middle Devonian, Canada and USA.

\section{Anastomopora sp.}

Material. - Ten fragmentary specimens, DGO 13037-13046. All specimens are recrystallized and partially silicified, producing evident loss of detail in both external and internal features. Sectioned specimens did not yield sections suitable for microscopic study of internal characters. Morphometric characters are provided in this description as a basic quantitative approach to the species, but it must be stressed that the bad preservation prevents accurate measurements. The description below is the result of partial observations of both external and internal characters in different specimens.

Description. - Exterior: Zoarium reticulate, shape unknown. Branches sinuous, robust, connected by short, wide dissepiments. Branch width 0.45-0-64 mm. Zooecia arranged in 3-4 rows, up to 6 prior to branch bifurcation and 2-3 rows after bifurcation. Apertures circular, inflected by minute radial styles producing lobulated aspect, peristome thick. Fenestrules oval, 0.88-1.16 mm long. Branch spacing $0.77-1.05 \mathrm{~mm}$. Sparse, irregularly spaced spine-like styles between zooecial apertures and on the reverse of branches. Distinct longitudinal ridge on the reverse surface of branches.

Interior: Autozooecial chambers elongate hexagonal. Heterozooecia not observed.

Remarks. - The studied material has been assigned to Anastomopora on the basis of the morphology of branches, presence of a longitudinal ridge on the reverse of branches, number of zooecial rows and shape of chambers, but the accessory pores characteristic of this genus have not been 
observed. Ernst et al. (2011) described Anastomopora adnata (Hall, 1883) from the Santa Lucía Formation, which is the lateral equivalent to the Moniello Formation on the southern slope of the Cantabrian Mountains. These authors did not report accessory pores in A. adnata, the material figured by them is morphologically comparable to that described herein, and also shows a blurry recrystallized aspect that contrasts with the good preservation of other species described in their work. Despite the apparent similarity, and consequently with the poor preservation of the Asturian specimens, it is convenient to keep this species in open nomenclature.

Occurrence. - Upper Emsian-lower Eifelian, Arnao, Cantabrian Zone, NW Spain.

\section{Palaeobiography of fenestrate bryozoans from the Moniello Formation}

Biogeographic studies are strongly conditioned by the accuracy in the identification of taxa, the uneven knowledge of the faunas from different regions of the world, the discontinuity of the fossil record and the degree of association of the studied faunas with facies. Palaeogeographic analyses of Palaeozoic bryozoans are significantly biased by these factors, and conclusions must be assumed as approaches subject to modifications according to the progress of knowledge. Updated taxonomic work has been carried out recently on the fenestrate bryozoans from the Moniello Formation; Suárez Andrés \& Wyse Jackson (2014) described the rare Ernstipora mackinneyi, Suárez Andrés \& Ernst (2015) reported thirteen species of Fenestellidae and eighteen more belonging to other families have been identified in the present work. These data provide a rather detailed insight into the morphological and taxonomic diversity developed by fenestrates from NW Spain during the transition between the Early and Middle Devonian.

Boucot et al. $(1967,1969)$ set the existence of three geographic realms during the Lower Devonian, based on the distribution of brachiopods: the Malvinokafric, the Eastern Americas and the Old World Realm. Oliver (1976) and Bigey (1980) used these divisions in their biogeographic studies of Devonian rugose corals and bryozoans, respectively. The upper Emsian-lower Eifelian Moniello Formation was deposited in a carbonate platform belonging to the Rhenish-Bohemian Province of the Old World Realm, at the septentrional margin of Gondwana.

Many fenestrate genera from the Moniello Formation are cosmopolite or have very wide distributions, indicating a low degree of provincialism. Spanish fenestrates clearly show Gondwanan affinities, but a few taxa shared with American faunas also occur. This fact has already been observed in other groups from the Devonian from NW Spain,
Table 16. Measurements of Polypora inusitata McKinney \& Kříž, 1986. Abbreviations as in Table 1.

\begin{tabular}{|c|c|c|c|c|c|c|}
\hline & $\mathrm{X}$ & SD & $\mathrm{CV}$ & MAX & MIN & $\mathrm{N}$ \\
\hline Branch thickness (mm) & 0.50 & 0.043 & 0.0018 & 0.59 & 0.36 & 30 \\
\hline Branch width (mm) & 0.55 & 0.053 & 0.0034 & 0.69 & 0.46 & 55 \\
\hline Fenestrule length (mm) & 1.74 & 0.310 & 0.0871 & 2.27 & 1.23 & 63 \\
\hline Fenestrule width (mm) & 0.58 & 0.142 & 0.0198 & 0.82 & 0.24 & 63 \\
\hline Dissepiment width (mm) & 0.23 & 0.031 & 0.0013 & 0.29 & 0.16 & 65 \\
\hline $\begin{array}{l}\text { Distance between branch } \\
\text { centres }(\mathrm{mm})\end{array}$ & 1.02 & 0.277 & 0.0662 & 1.38 & 0.13 & 76 \\
\hline $\begin{array}{l}\text { Aperture spacing across } \\
\text { branch }(\mathrm{mm})\end{array}$ & 0.25 & 0.022 & 0.0009 & 0.33 & 0.20 & 103 \\
\hline $\begin{array}{l}\text { Aperture spacing along } \\
\text { branch }(\mathrm{mm})\end{array}$ & 0.31 & 0.037 & 0.0012 & 0.39 & 0.23 & 91 \\
\hline Aperture width (mm) & 0.11 & 0.016 & 0.0002 & 0.17 & 0.09 & 117 \\
\hline $\begin{array}{l}\text { Maximal chamber width } \\
(\mathrm{mm})\end{array}$ & 0.15 & 0.016 & 0.0004 & 0.19 & 0.11 & 114 \\
\hline Chamber length (mm) & 0.24 & 0.037 & 0.0011 & 0.31 & 0.18 & 114 \\
\hline Chamber depth (mm) & 0.17 & 0.012 & 0.0004 & 0.20 & 0.14 & 24 \\
\hline $\begin{array}{l}\text { Angle transverse } \\
\text { wall-basal plate } \\
\text { (degrees) }\end{array}$ & 57.02 & 5.43 & 20.56 & 70.22 & 40.64 & 17 \\
\hline
\end{tabular}

such as rugose (Soto 1979, 1982) and tabulate corals (Fernández-Martínez 1993), which show a high degree of cosmopolitism in the Emsian, or trilobites (Arbizu 1978). Recent studies (May 2005, Rachebouf et al. 2012) report affinities between tabulate corals from NW Spain and Bolivia. Ernst (2010, 2011), Ernst et al. (2011) and Ernst \& Buttler (2012) reported bryozoan species previously known from North America in the Emsian and Eifelian of NW Spain. Affinities of the fenestrates from the Moniello Formation with Laurentian forms are supported by the presence of Fenestella aff. parallela, F. vera and Ptylopora aff. nodosa (Suárez Andrés \& Ernst 2015), but most significantly by Fenestrapora occidentalis. According to the reported occurrences, Fenestrapora was a shortlived genus (upper Emsian to Givetian) with few known species, but its geographic distribution was relatively wide, ranging from US to Spain, Germany and Azerbaijan (Ernst 2016). The number of species shared with the Devonian of North America is probably underestimated, partly due to the great number of taxa described in early taxonomic works, which largely lack information on their inner morphology. Most of these taxa have not been restudied according to updated procedures, what hampers reasonably accurate assessment of faunal similarities.

Among the Old World regions, the fenestrate bryozoans of the Moniello Formation show the highest affinities with the fauna from the Lower Devonian of the Prague Basin, Czech Republic, as detailed below, but apparently lower numbers of species shared with intermediate regions should be discussed. 
Descriptions on Devonian fenestrates from France generally provide limited information, which hinders species-level comparisons; nevertheless, and beyond cosmopolitan genera, a few genera with less widespread distributions in the Lower-Middle Devonian are shared with the Moniello Formation. Bigey (1972a, b) reported the presence of Ptylopora and Utropora from the Armorican Massif, the latter being a relatively restricted genus identified in Spain (herein), Bohemia, Transcaucasia (Morozova \& Lavrentjeva 1998) and the Lower Carboniferous of China (Xia \& Lu 1986). Bigey \& Feist (1975) described silicified upper Emsian fenestrates from the Armorican Massif including Fenestella, Hemitrypa, Ptylopora and Penniretepora, but species are not common with the Spanish fauna. Babin et al. (1980) reported species of Hemitrypa, Semicoscinium or Fenestella (sensu lato) in open nomenclature, all three widely distributed genera, but according to the illustrations and morphometric characters provided by the authors, these species have not been found in the Moniello Formation. Babin et al. (1981) illustrate transverse sections of bryozoan species assigned to undetermined fenestrates, which at present can be undoubtedly assigned to the Semicosciniidae. The species named as "Fenestellid 1" (Babin et al. 1981, pl. 13, figs 1-2) might belong to Fenestrapora. No rigorous identification can be made for "Fenestellid 2", and "Fenestellid 3" could be assigned to Bigeyina on the basis of the bifurcation of the keel top (Babin et al. 1981, pl. 13, fig. 4). This would be reasonable, as Bigeyina has been reported from Bohemia, Germany and Spain.

Little is known about the Devonian fenestrates of Belgium. Dessilly \& Kräusel (1962) described two species in open nomenclature belonging to Hemitrypa and Semicoscinium. Dessilly \& Kräusel (1963) named the new genus Speotrypa, which nowadays is considered a junior synonym of Semicoscinium (McKinney 2009). Dessilly (1967) identified for the first time a Devonian phylloporinid species in Belgium, "Phylloporina" devonica, later assigned to Bashkirella by Lavrentjeva (1985) and found in the Rhenish Slate Massif (Germany) by Ernst \& Schroeder (2007).

The Devonian fenestrate faunas from Germany share several genera with the Moniello Formation, most of them having little palaeobiogeographic significance due to their wide geographic and stratigraphic distributions, except for Fenestrapora and Bigeyina. The latter was first described in the Middle Devonian of Germany (Bornemann 1884) but the earliest species have been found in the Lower Devonian of Bohemia (McKinney \& Kříž 1986). Besides these two regions and the possible case in France discussed above, Bigeyina has only been reported from the Emsian-lower Eifelian of NW Spain (Suárez Andrés 1998, Ernst 2012, Suárez Andrés \& Wyse Jackson 2015, herein). After the transfer and restitution of $B$. bohemica
Prantl, 1932 as the type species of the genus Pseudoisotrypa (Suárez Andrés \& McKinney 2010), new taxa described in Spain, including B. cantabrica and B. spinosa from the Moniello Formation, increased the known composition of Bigeyina from four to seven species. This short-lived genus underwent increasing diversity and disparity in the upper Emsian, as indicated by the number of species present in Spain and the occurrence of structural innovations, i.e. the bridged keels and the composite tubular morphology developed by $B$. ibera.

The revision made by McKinney \& Křriž (1986) of the Bohemian fauna described by Barrande (Počta 1894) and Prantl (1932) is a thorough systematic work that stressed the diversity of the Lower Devonian fenestrates from the Prague Basin. Clear affinities have been established between the Bohemian and Spanish fenestrates on the basis of this study. Significant similarities comprise seven shared species, most of them belonging to genera with wide geographic and stratigraphic distributions, such as Polypora inusitata or Spinofenestella strigilla, but also the common presence of Bigeyina, Utropora and Filites, which have shorter distributions. Until this research Filites, originally described from Bohemia, was a monotypic genus, if one accepts that Morozova (2001) erroneously included species with triangular chambers previously assigned to Penniretepora from different stages and regions. Unlike these taxa, which the current authors consider to belong to Penniretepora, the Spanish F. robustus described here shares with the type species, $F$. bohemicus, diagnostic vestigial, backward recurved pinnae bearing zooecia morphologically different from those on the main branch.

Few affinities have been observed between fenestrates from the Moniello Formation and eastern faunas beyond Bohemia, except for the common presence of widespread genera. Only two common species have been found, Fenestella vera Ulrich, 1890 and Rectifenestella pioneri (Krasnopeeva, 1935). The former is a widely distributed species that has been identified in the Middle Devonian Hamilton Group, Buffalo, USA (Ulrich 1890), Lake Winnipegosis, Canada (Whiteaves 1892), and the Givetian of Mongolia (Nekhoroshev 1926), Altai (Krasnopeeva 1935, Volkova 1974) and Kuznets Basin (Morozova 1960). Rectifenestella pioneri has been reported from the Middle Devonian of Altai (Krasnopeeva 1935, Nekhoroshev 1948, Volkova 1974) and Kuznets Basin (Morozova 1960).

Though palaeogeographic links with other regions are supported by the affinities discussed above, the bryozoans of the Order Fenestrata of the Moniello Formation comprise fourteen new species (Suárez Andrés \& Wyse Jackson 2014, Suárez Andrés \& Ernst 2015, herein), which represent almost $50 \%$ of the identified fauna. It might be interpreted as the evidence of a high degree of provincialism, but this apparent endemism is possibly overestimated, 
due to the confluence of circumstances such as the degree of knowledge of the regions subject to comparison. The active research on Devonian bryozoans carried out by several authors in recent years continuously increases the number of new taxa, but also the geographic distributions of some previously known species; much work has to be done in the field and restudying historical collections, so the present knowledge is not likely to become balanced in a short term. Among the new taxa, five species represent the earliest and westernmost occurrence of their "Lazarus genera", and significantly increase their geographic and stratigraphic distributions. This fact has also been observed in rugose corals belonging to the Cyathaxonia fauna, some of which occurred for the first time in the Lower Devonian of the Cantabrian Range and later expanded becoming even cosmopolite taxa (Soto 1982). In his study of the Devonian Cyathaxonia fauna, Wrzolek (2002) named "silent taxa" those supposedly monophyletic that show significant gaps in their total distributions. The author considered two possible causes for these gaps, either lack of knowledge of the taxon in a given interval ("Lazarus taxa"), or extinction and further occurrence of a misidentified "Elvis taxon".

Bashkirella has been reported from the upper Eifelian of Belgium, Germany and Armenia, as well as from the Carboniferous of Russia and China and early Permian of Russia. Paraseptopora has a relatively complete stratigraphic record from the Famennian (Upper Devonian) of Kazakhstan up to the Permian in different regions of the former USSR (Morozova 2001), but no species have been reported from Central or Western Europe. Kalvariella is a rare genus with only five known species including $K$. antiqua sp. nov.; previously reported species occurrences range from the Carboniferous of the Arctic region of Kolyma to the Permian of Thailand (Sakagami 1970, Morozova 2001), so K. antiqua sp. nov. greatly expands its distribution and stratigraphic range downwards into the Devonian. The occurrence of Permofenestella, a Permian genus from Russia and Mongolia, in the Mississippian (lower Carboniferous) of Ireland (Wyse Jackson 2001) seems to reflect the same downward stratigraphic trend. As a possible explanation to this gap, the delicate structure of Kalvariella possibly reduced its preservation potential when compared to reticulate forms; furthermore, this genus closely resembles Penniretepora, and can be easily misidentified if multiserial pinnae cannot be clearly observed. Cavernella also shows a wide gap in its stratigraphic record between the Lower Devonian of NW Spain and the Carboniferous of Hungary (Zágoršek 1993). It can be reasonably assumed that it should occur in intermediate sections, though specimens can be erroneously assigned to Rectifenestella if heterozooecia are not noticed in an appropriate tangential section.

Early occurrences of fenestrate genera have been reported in the Emsian of NW Spain beyond the Moniello
Formation. Ernst (2012) described Fenestrapora occidentalis from the Emsian of León, increasing the stratigraphic distribution of the species and the genus at a time: Fenestrapora had been previously described only from the Middle Devonian of USA, Transcaucasia and Germany. Ernst (2012) also reported two new species of Prolixicella that are the earliest known representatives of their genus and the only ones of Early Devonian age. Ernst \& Königshof (2010) named the new genus Dissotrypa from the Givetian (Middle Devonian) of West Sahara, and Ernst (2012) defined two new species from the Emsian of León, Spain, thus placing in the Early Devonian the first appearance of both Dissotrypa and the Family Septatoporidae.

The fenestrate fauna of the Moniello Formation is highly diverse but not only new taxa occurred; uncommon growth habits have been reported that could be framed in a peak of disparity within the evolution of fenestrates, as reported by Suárez Andrés \& Wyse Jackson (2015). Besides zoarial morphology, other innovations seem to indicate an important episode of the evolutionary history of fenestrate bryozoans occurring in the Lower Devonian: the presence of Cavernella and Fenestrapora in this Emsian-Eifelian fauna, together with the occurrence of Dissotrypa and Iberofenestella in the Emsian of León (Ernst 2012) point to a high diversification of heteromorphs, while most of these structures are known to occur in Late Palaeozoic taxa. Dissotrypa is also meaningful due to the development of proximal pores (McKinney \& Wyse Jackson 2015, pp. 49-51), a puzzling structure that has been described in other Carboniferous (Engel 1975, Tavener-Smith 1971) and Permian forms (Gautier et al. 2013).

\section{Conclusions}

Eighteen species of non-Fenestellidae fenestrate bryozoans, nine of which are new, have been described from the upper Emsian-lower Eifelian Moniello Formation. Considering the previously reported ones belonging to the Fenestellidae, (Suárez Andrés \& Wyse Jackson 2014, Suárez Andrés \& Ernst 2015), the known fenestrate fauna of the Moniello Formation is composed of thirty-two species, thus representing a highly diverse group.

The fenestrate bryozoans of the Moniello Formation show strong affinities with the Early Devonian fauna from Bohemia within the Old World Realm, but species shared with the Eastern America Realm also exist.

In contrast with Bohemia, affinities of the Moniello Formation fenestrates with those from other close regions within the Old World Realm are possibly underestimated due to insufficient knowledge of these local faunas.

Among the high diversity of the Moniello Formation fauna, several species represent the earliest reported record 
of their genera and their most western distribution, including Bashkirella, Calvariella, Kavernella and Paraseptopora. Hitherto all of them mostly were composed of Carboniferous and Permian species, and their presence in the Devonian of Spain significantly extends their stratigraphic and geographic distributions.

Innovative features of some fenestrates of the Moniello Formation, together with those of contemporary faunas from other regions, depict a period of high diversity and disparity within the evolutionary history of the Order Fenestrata that took place in the Emsian-Eifelian.

\section{Acknowledgements}

This research received support from the SYNTHESYS Project http://www.synthesys.info/ which is financed by European Community Research Infrastructure Action under the FP7 "Capacities" Programme, through a grant awarded to J.S. in 2012 (GB-TAF 2129) to visit the Natural History Museum, London. Cova González is thanked for assisting with graphic work. We thank the reviewers for their valuable and constructive comments. Andrej Ernst (Hamburg) is thanked for his help providing Russian references.

\section{References}

Alonso, J.L., Marcos, A. \& SuÁrez, A. 2009. Paleogeographic inversion resulting from large out of sequence breaching thrust: The León Fault (Cantabrian Zone, NW Iberia). A new picture of the external Variscan thrust belt in the Ibero-Armorican Arc. Geologica Acta 7, 451-473.

Arbizu, M. 1978. Trilobites Synphoriidae del Devónico de la Cordillera Cantábrica: Malladiinae nov. subfam. Trabajos de Geología 10, 37-65.

Arbizu, M. \& Méndez-Bedia, I. 2006a. Patrimonio natural y cultural de Castrillón, Asturias, 18. In García Ramos, J.C. et al. (eds) Resúmenes VII Reunión de la Comisión para el Patrimonio Geológico. Colunga. Sociedad Geológica de España.

Arbizu, M. \& MÉndez-BediA, I. 2006b. El Patrimonio Natural y Cultural de Castrillón (Asturias): Geología, Fósiles e Historia Minera. Trabajos de Geología 26, 73-91.

Arbizu, M., García-Alcalde, J.L., García-López, S., Méndez-Bedia, I., SÁnchez de Posada, L.C., Soto, F.M., Truyols, M., Truyols, J., Álvarez, F., Méndez, C. \& MenÉndeZ, J.R. 1979. Biostratigraphical study of the Moniello Formation (Cantabrian Mountains, Asturias, NW Spain). A contribution to the Lower/Middle Devonian boundary problem. Geologica et Palaeontologica 13, 103-124.

Arbizu, M., Méndez-Bedia, I. \& Soto, F. 1991. Desarrollo arrecifal en el Devónico Inferior de los alrededores de Arnao, Costa Asturiana. Guía de campo de la excursión $n^{\circ} 1$, VII Jornadas de Paleontología. Universidad de Oviedo, 1-30.
Astrova, G.G. \& Morozova, I.P. 1956. New order of Paleozoic Bryozoa. Paleontologicheskii Zhurnal 1, 22-31. [in Russian] Babin, C., Bigey, F., Brice, D., Carls, P., Deunff, J., Goujet, D., Heddebaut, C., Jahnke, H., Lafuste, J., Lardeux, H., Lejal-Nicol, A., Le Menn, J., Moreau-Benoit, A., Morzadec, P., Paris, F., Pelhate, A., Plusquellec, Y., Poncet, J., Racheboeuf, P. \& Weyant, M. 1980. Les Schistes et Calcaires de l'Armorique (Devonien Inférieur, Massif Armoricain). Sédimentologie, paléontologie, stratigraphie. Bryozoans, 181-193. In Plusquellec, Y. (ed.) Mémoires de la Société Géologique et Minéralogique de Bretagne 23.

Babin, C., Bigey, F., Brice, D., Copper, P., Coquel, R., Goujet, D., Heddebaut, C., Jahnke, H., Lardeux, H., Le Menn, J., Melou, M., Morzadec, P., Paris, F., Pehate, A., Plusquellec, Y., Rachebouef, P. \& Weyant, M. 1981. La Tranchée de La Lezais. Emsien Supérieur du Massif Armoricain. Sédimentologie, paléontologie, stratigraphie. Bryozoans, 109-124. In Morzadec, P., Paris, F. \& Rachebouef, P. (eds) Mémoires de la Société Géologique et Minéralogique de Bretagne 24.

Bigey, F.P. 1972a. Présence de Utropora aff. nobilis (Bryozoaire Cryptostome) dans le Dévonien du Sud Est du Massif Armoricain. Bulletin de la Société géologique de France XIV, 315-319. DOI 10.2113/gssgfbull.S7-XIV.1-5.315

Bigey, F.P. 1972b. Présence de Ptilopora aff. bohemica (Bryozoaire Cryptostome) dans le Dévonien du Sud Est du Massif Armoricain. Bulletin de la Société d'Etudes Scientifiques de l'Anjou VIII, 15-22.

Bigey, F.P. 1980. Biogeography of Devonian Bryozoa, 9-23. In Nielsen, C., LaRwood, G.P. (eds) Bryozoa: Ordovician to Recent. Olsen \& Olsen, Fredensborg.

Bigey, F.P. \& Feist, R. 1975. Cryptostomata (Bryozoaires) des "Calcaires à Polypiers silicieux" (Dévonien Inferieur de la Montagne Noire, France Meridionale). Note preliminaire, 257-271. In Pouyet, S. (ed.) Bryozoa, 1974. Documents des Laboratoires de Géologie de la Faculté des Sciences de Lyon H.S. 3(2).

BorG, F. 1926. Studies on Recent cyclostomatous Bryozoa. Zoologiska Bidrag från Uppsala 10, 181-507.

Bornemann, J.G. 1884. Cyclopelta winteri, eine Bryozoe aus dem Eifeler Mitteldevon. Zeitschrift der Deutschen Geologischen Gesellschaft 36, 864-865.

Boucot, A.J., Johnson, J.G. \& Talent, J.A. 1967. Lower and Middle Devonian faunal provinces based on Brachiopoda, 1239-1254. In OswaLd, D.H. (ed.) International Symposium on the Devonian System 2.

Boucot, A.J., Johnson, J.G. \& TAlent, J.A. 1969. Early Devonian brachiopod zoogeography. Geological Society of America Special Papers 119, 1-113.

Cомте, P. 1936. Le Dévonien Inférieur du Léon. Comptes Rendus de l'Academie des Sciences de Paris 202, 771-773.

DessiLly, E. 1967. Les bryozoaires dévoniens de la Belgique: la presence du genre "Phylloporina" dans le Couvinien de la Belgique. Bulletin de l'Institut Royal des Sciences Naturelles de Belgique 43(3), 1-6.

Dessilly, E. \& Kräusel, W. 1962. Les Bryozoaires Dévoniens de 
la Belgique. La découverte des genres Hemitrypa et Semicoscinium dans le Dévonien de la Belgique. Bulletin de l'Insitut royal des Sciences Naturelles de Belgique 38(20), $1-5$.

Dessilly, E. \& Kräusel, W. 1963. Les Bryozoaires Dévoniens de la Belgique. Speotrypa, nouveau genre du Couvinien de la Belgique. Bulletin de l'Insitut royal des Sciences Naturelles de Belgique 39(31), 1-13.

EhrenberG, C.G. 1831. Symbolae Physicae, seu Icones et descriptiones. Pars Zoologica, IV, Animalia Evertebrata exclusis Insectis. Berolini ex Officina Academica, Berlin.

Elias, M.K. \& CondRA, G.E. 1957. Fenestella from the Permian of West Texas. The Geological Society of America Memoir 70, $1-158$.

ENGEL, B.A. 1975. A new ? bryozoan from the Carboniferous of Eastern Australia. Palaeontology 18(3), 571-605.

ERnst, A. 2010. Trepostome bryozoans from the Lower-Middle Devonian of NW Spain. Rivista Italiana di Paleontologia e Stratigrafia 116, 283-308.

ERnst, A. 2011. Cryptostome (ptilodictyine and rhabdomesine) Bryozoa from the Lower Devonian of NW Spain. Palaeontographica Abteilung A: Paläozoologie-Stratigraphie 293, 147-183.

ERnst, A. 2012. Fenestrate bryozoan fauna of the Lower-Middle Devonian of NW Spain. Neues Jahrbuch für Geologie und Paläontologie Abhandlungen 264(3), 205-247.

ERnst, A. 2013. Diversity dynamics and evolutionary patterns of Devonian Bryozoa. Palaeobiodiversity and Palaeoenvironments 93, 45-63.

Ernst, A. 2016. Fenestrapora (Fenestrata, Bryozoa) from the Middle Devonian of Germany. Paläontologische Zeitschrift 90, 19-32. DOI 10.1007/s12542-016-0284-x

ERnst, A. \& Buttler, C. 2012. Cystoporate bryozoans from the Lower-Middle Devonian of NW Spain. Neues Jahrbuch für Geologie und Paläontologie, Abhandlungen 263, 261-285.

ERnst, A. \& Königshof, P. 2010. Bryozoan fauna and microfacies from a Middle Devonian reef complex (Western Sahara, Morocco). Abhandlungen der Senckenberg Gesellschaft für Naturforschung 568, 1-191.

ERnst, A. \& Schroeder, S. 2007. Stenolaemate bryozoans from the Middle Devonian of the Rhenish Slate Massif (Eifel, Germany). Neues Jahrbuch für Geologie und Paläontologie, Abhandlungen 246(2), 205-233.

Ernst, A. \& Winkler Prins, C.F. 2008. Pennsylvanian bryozoans from the Cantabrian Mountains (northwestern Spain). Scripta Geologica 137, 1-123.

Ernst, A., Dorsch, T. \& Keller, M. 2011. A bryozoan fauna from the Santa Lucia Formation (Lower-Middle Devonian) of Cantabrian Mountains, NW Spain. Facies 57, 301-329.

Fernández, L.P., Nose, M., Fernández-Martínez, E., MéndezBedia, I., SchröDer, S. \& Soto, F. 2006. Reefal and mud mound facies development in the Lower Devonian La Vid Group at the Colle outcrops (León province, Cantabrian Zone, NW Spain). Facies 52, 307-327.

DOI 10.1007/s10347-005-0042-0

FERnÁNDEZ-MartínEZ, E. 1993. Tabulados y Chaetétidos de las
Formaciones Moniello-Santa Lucía y Candás-Portilla (Devónico, Cordillera Cantábrica, NO de España). 420 pp. Ph.D. thesis, Universidad de Oviedo, Spain.

Fernández-Martínez, E., Fernández, L.P., García-Alcalde, J., MéndeZ-Bedia, I. \& Soto, F. 2006. Excursión A. El Devónico arrecifal de la Zona Cantábrica. XXII Jornadas de la Sociedad Española de Paleontología, 1-62.

García-López, S. \& SANZ-López, J. 2002. Devonian to Lower Carboniferous conodont biostratigraphy of the Bernesga Valley section (Cantabrian Zone, NW Spain), 163-205. In García-López, S. \& Bastida, F. (eds) Palaeozoic conodonts from northern Spain. $8^{\text {th }}$ International Conodont Symposium held in Europe. Publicaciones del Instituto Geológico y Minero de España, Cuadernos del Museo Geominero 1.

García-López, S., SAnz-López, J. \& Sarmiento, G.N. 2002. The Palaeozoic succession and conodont biostratigraphy of the section between Cape Peñas and Cape Torres (Cantabrian coast, NW Spain), 125-161. In GARcía-LóPEZ, S., BASTIDA, F. (eds) Palaeozoic conodonts from northern Spain. $8^{\text {th }}$ International Conodont Symposium held in Europe. Publicaciones del Instituto Geológico y Minero de España, Cuadernos del Museo Geominero 1.

Gautier, T.G., Wyse Jackson, P.N. \& McKinney, F.K. 2013. Adlatipora, a distinctive new acanthocladiid bryozoan from the Glass Mountains, Texas, USA, and its bearing on fenestrate astogeny and growth. Journal of Paleontology 87 , 444-455. DOI 10.1666/12-128.1

GoRJunOva, R.V. 1966. On a new genus of the bryozoan Family Phylloporinidae. Paleontologicheskii Zhurnal 2, 127-130. [in Russian]

HALL, J. 1883. Bryozoans of the Upper Heldelberg and Hamilton groups. Transactions of the Albany Institute 10, 145-197.

Hall, J. 1884. Bryozoa (Fenestellidae) of the Hamilton Group. $36^{\text {th }}$ Annual report on the New York State Museum of Natural History, 57-72.

Hall, J. 1885. Bryozoans of the Hamilton Group. Report of the State geologist for the year 1883, 5-61.

Kopajevich, G.V. 1975. Silurian bryozoans from Estonia and Podolia. Trudy Paleontologicheskogo Instituta Akademiya Nauk SSSR 171, 1-155. [in Russian]

Krasnopeeva, P.S. 1935. Bryozoans of the Middle and Upper Devonian of Altai. Materiali po Geologii Zapadno-Sibirskogo Kraya 2, 43-84. [in Russian]

Lavrentueva, V.D. 1979. Phylloporinina - A new Suborder of Paleozoic Bryozoa. Paleontological Zhurnal 1, 59-68.

LavrentjeVA, V.D. 1985. Bryozoans of the suborder Phylloporinina. Trudy Paleontologicheskogo Instituta Akademiya Nauk SSSR 214, 1-101. [in Russian]

Lisitsyn, D.V. 1991. New Permian bryozoans from the Northern Urals. Paleontological Journal 25(4), 88-99.

May, A. 2005. A new Parastriatopora species (Anthozoa, Tabulata) from the Lower Devonian of Colle (Spain, Cantabrian Mountains). Bulletin of Geosciences 80, 287-290.

M'Coy, F. 1844. A synopsis of the character of the Carboniferous Limestone fossils of Ireland. 207 pp. University Press, Dublin. DOI 10.5962/bhl.title.11559 
McKinney, F.K. 1998. Avicularia-like structures in a Paleozoic fenestrate bryozoan. Journal of Paleontology 72(5), 819-826. DOI 10.1017/S0022336000027177

McKinney, F.K. 2009. Bryozoan-hydroid symbiosis and a new ichnogenus, Caupokeras. Ichnos 16, 193-201.

DOI 10.1080/10420940802686160

McKinney, F.K. \& KŘíž, J. 1986. Lower Devonian Fenestrata (Bryozoa) of the Prague Basin, Barrandian Area, Bohemia, Czechoslovakia. Fieldiana Geology n. s. 15, 1-90.

McKinney, F.K. \& Wyse Jackson, P.N. 2015. Part G, Revised, Volume 2, Chapter 8A: Order Fenestrata: Morphology and growth. Treatise Online 66, 1-91.

Méndez-Bedia, I. 1976. Biofacies y litofacies de la Formacion Moniello-Santa Lucía (Devónico de la Cordillera Cantábrica, NO de España). Trabajos de Geología 9, 1-93.

Méndez-Bedia, I. \& Soto, F. 1984. Paleoecological succesion in a Devonian organic buildup (Moniello Fm., Cantabrian Mountains, NW NW Spain). Geobios 8, 151-157. DOI 10.1016/S0016-6995(84)80168-0

Méndez-Bedia, I., Soto, F. \& Fernández-Martínez, E. 1994. Devonian reef types in the Cantabrian Mountains, (NW Spain) and their faunal composition. Courier Forschungsinstitut Senckenberg 172, 161-183.

Morozova, I.P. 1960. Devonian bryozoans from the Kuznets and Minusin Basins. Trudy Paleontologicheskogo Instituta, Akademiya Nauk SSSR 86, 1-172. [in Russian]

Morozova, I.P. 1962. Systematics and phylogeny of Fenestelloidea. Paleontologicheskii Zhurnal 4, 104-115. [in Russian]

Morozova, I.P. 1970. Late Permian bryozoans. Trudy Paleontologicheskogo Instituta, Akademiya Nauk SSSR 122, 1-347. [in Russian]

Morozova, I.P. 1987. Morphogenesis, system and colonial integration in Bryozoa of the Order Fenestrida. Trudy Paleontologicheskogo In-ta Akademii Nauk SSSR 222, 70-88. [in Russian]

Morozova, I.P. 2001. Bryozoans of the Order Fenestellida. Trudy Paleontologicheskogo Instituta 277, 1-176. [in Russian]

Morozova, I.P. \& LavrentJeVA, V.D. 1998. New bryozoans of the Family Semicosciniidae. Paleontological Journal, 32(2), 154-162.

Nekhoroshev, V.P. 1926. Bryozoans of the Middle Devonian of NE Mongolia, with description of a microscopic method of study for fenestellids. Trudy Geologicheskiy Muzei Akademii Nauk SSSR 1, 1-28. [in Russian]

Nekhoroshev, V.P. 1948. Bryozoans of the Devonian of Altai. Paleontologiya SSSR 3(2-1). Izdatel'stvo Akademii Nauk SSSR Paleontologicheski Institut, 1-172. [in Russian]

Nekhoroshev, V.P. 1977. Devonian bryozoans of Kazakhstan. Ministerstvo Geologii SSSR Vsesoyuznyy Ordena Lenina Nauchno-Issledovatel'skiy Geologicheskiy Institut Trudy (n.s.) 186, 1-192. [in Russian]

Nikiforova, A.I. 1938. Types of Carboniferous Bryozoa from the European part of the USSR. Izdatel'stvo Akademii Nauk SSSR, Paleontology of USSR 4, part 5(1), 1-290. [in Russian]
Nikiforova, A.I. 1939. New species of the Upper Palaeozoic bryozoans of the Bashkirian Urals. Trudy Neftyanogo Geologo-razvedochnogo Instituta, (A) 115, 70-102. [in Russian]

Oliver, W.A. 1976. Biogeography of Devonian rugose corals. Journal of Paleontology 50, 365-373.

OrbignY, M.A. D' 1849. Descriptions de quelques genres nouveaus de mollusques bryozoaires. Revue et magasin de Zoologie 1(2), 499-501.

Pérez-Estaún, A., Bastida, F., Alonso, J.L., Marquínez, J., Aller, J., Álvarez-Marrón, J., Marcos, A. \& Pulgar, J.A. 1988. A thin-skinned tectonics model for an arcuate fold and thrust belt: the Cantabrian Zone. Tectonics 7, 517-538. DOI 10.1029/TC007i003p00517

Pérez-Estaún, A., Bea, F., Bastida, F., Marcos, A., Martínez-Catalán, J.R., Martínez-Poyatos, D., Arenas, F., Díaz-García, F., Azor, A., Simancas, J.F., GonzálezLodeIRo, F. 2004. La Cordillera Varisca Europea. El Macizo Ibérico, 19-230. In VerA, J.A. (ed.) Geología de Espańa, SGE - IGME, Madrid.

PhILlips, J. 1836. Illustrations of the geology of Yorkshire. 2. The Mountain Limestone District, 253 pp. John Murray, London.

Plamenskaya, A. 1991. Bryozoans of the Lower and Upper Devonian of Kazakhstan, 91-111. In Dubatolov, V.N. \& Stukalina, G.A. (eds) Biostratigraphy of the Lower and Upper Devonian of the Djulgaro-Balkhash provinces. Akademiya Nauyk SSSR, Sibirskoe Otdelenie, Institut Geologii I Geofiziki, Novosibirsk. [in Russian]

PočTA, P. 1894. Systême Silurien du Centre de la Bohême par Joachim Barrande. Première Partie: Recherches Paleontologiques VIII (1). 230 pp. Privately published, Prague \& Paris.

Prantl, F. 1932. Revision of the fenestellids from the Devonian of Bohemia. Palaeontographica Bohemiae XV, 1-70.

Prout, H.A. 1859. Third series of descriptions of Bryozoa from the Palaeozoic rocks of western states and territories. Transactions of St. Louis Academy of Sciences (S. 3), 443-452.

Racheboeuf, P.R., Casier, J.G., Plusquellec, Y., Toro, M., Mendoza, D., Pires de Carvalho, M.G., Le Herissé, A., Paris, F., Fernández-Martínez, E., Tourneur, F., Broutin, J., Crasquin, S. \& Janvier, P. 2012. New data on the Silurian-Devonian paleontology and biostratigraphy of Bolivia. Bulletin of Geosciences 86, 1-46.

DOI 10.3140/bull.geosci.1248

Sakagami, S. 1970. Addition to the Permian Bryozoa from Ko Muk, peninsular Thailand. Geology and Palaeontology of Southeast Asia 8, 43-68.

SÁnchez de la Torre, L. \& Manjón, M. 1976. Facies de la Caliza de Moniello en la zona de Arnao, Asturias. Trabajos de Geología 8, 109-129.

Shulga-nesterenko, M.I. 1955. Carboniferous Bryozoa of the Russian Platform. Trudy Paleontologicheskogo Instituta, Akademiya Nauk SSSR 57, 1-191. [in Russian]

Simpson, G.B. 1897. A handbook of the genera of the Norht American Paleozoic Bryozoa. $14^{\text {th }}$ Annual report of the state geologist (of New York) for the year 1894, 407-608. 
Soto, F. 1979. Considérations paléobiogéobraphiques sur les Streptelasmatina (Coelenterata, Rugosa) solitaires du Dévonien des Monts Cantabriques (NE de l'Espagne). Geobios 3, 399-409. DOI 10.1016/S0016-6995(79)80119-9

Soto, F. 1982. Plerophyllidae y Pentaphyllidae (Coelenterata, Rugosa) del Devónico de la Cordillera Cantábrica (NO de España). Trabajos de Geología 12, 49-61.

Soto, F. \& MÉndez-Bedia, I. 1985. Estudio de una asociación coral rugoso-estromatopórido en el arrecife de Arnao (Fm. Moniello, Asturias, NO de España). Trabajos de Geología 15, 203-209.

Soto, F., Méndez-Bedia, I. \& Fernández-Martínez, E. 1994. Construcciones arrecifales del Devónico de la Cordillera Cantábrica (NO de España). Revista Española de Paleontología 9, 29-36.

SuÁreZ Andrés, J.L. 1998. Briozoos Fenestrados de la Formación Moniello (Devónico) en el área de Arnao (Asturias, España) I: Fenestellidae. Revista Española de Paleontología 13(2), 187-196.

SuÁrez ANDRÉs, J.L. 1999. Briozoos Fenestrados de la Formación Moniello (Devónico) en el área de Arnao (Asturias, España) II: Septoporidae, Polyporidae y Acanthocladiidae. Revista Española de Paleontología Numero Extraordinario Homenaje al Profesor Truyols, 185-193.

SuÁREZ ANDRÉs, J.L. 2014. Bioclaustration in Devonian fenestrate bryozoans. The ichnogenus Caupokeras McKinney, 2009. Spanish Journal of Paleontology 29, 5-14.

SuÁreZ AndRÉs, J.L. \& ERnst, A. 2015. Lower-Middle Devonian Fenestellidae (Bryozoa) of NW Spain: Implication for fenestrate paleobiogeography. Facies 61(415), 1-29. DOI 10.1007/s10347-014-0415-3

SuÁrez Andrés, J.L. \& GonZÁlez Álvarez, M.C. 1999. Nuevos datos sobre los briozoos Devónicos de la Zona Cantábrica, 643-646. In RÁBAno, I. (ed.) Actas de las XV Jornadas de la Sociedad Española de Paleontología y Simposios de los proyectos PICG 393, 410 y 421, Colección Temas Geológico-Mineros 26. Instituto Tecnológico Geominero de España, Madrid.

SuÁrez Andrés, J.L. \& McKinney, F.K. 2010. Revision of the Devonian Fenestrate bryozoan genera Cyclopelta Bornemann, 1884 and Pseudoisotrypa Prantl, 1932, with description of a rare growth habit. Revista Española de Paleontología 25(2), $123-138$.

SuÁreZ ANDRÉs, J.L. \& Wyse Jackson, P.N. 2014. Ernstipora mackinneyi, a new unique fenestrate bryozoan genus and spe- cies with an encrusting growth habit from the Emsian (Devonian) of NW Spain. Neues Jahrbuch für Geologie und Paläontologie Abhandlungen 271(3), 229-242.

DOI 10.1127/0077-7749/2014/0384

SuÁrez Andrés, J.L. \& Wyse Jackson, P.N. 2015. Feeding currents: a limiting factor for disparity of Palaeozoic fenestrate bryozoans. Palaeogeography, Palaeoclimatology, Palaeoecology 433, 219-232. DOI 10.1016/j.palaeo.2015.05.015

SuÁrez Andrés, J.L., Wyse Jackson, P.N. \& Sendino, C. 2014. First record of a Palaeozoic fenestrate with a secondary zooid-bearing meshwork. Studi Trentini delle Scienze Naturali 94, 241-247.

Tavener-Smith, R. 1971. Polypora stenostoma, a Carboniferous bryozoan with cheilostomatous features. Palaeontology 14(1), $178-187$.

UlRICH, E.O. 1890. Palaeozoic Bryozoa. Illinois Geological Survey Bulletin 8, 283-688.

Vine, G.R. 1880. On the Carboniferous Polyzoa. Geological Magazine II (VII), 501-512.

DOI 10.1017/S0016756800151532

Volkova, K.N. 1974. Devonian Bryozoa from the Southeast Altai. Trudy Institut Geologii i Geofiziki 199, 1-182. [in Russian]

WhiteAves, J.F. 1892. The fossils of the Devonian rocks of the islands, shores or immediate vicinity of Lakes Manitoba and Winnipegosis. Contributions to Canadian Palaeontology 1(4), 255-359.

WrzoleK, T. 2002. Devonian history of diversity of the rugosan Cyathaxonia fauna. Acta Palaeontologica Polonica 47, 397-404.

Wyse Jackson, P.N. 2001. Permofenestella Morozova, 1974 (Fenestrata, Bryozoa): first record from the Lower Carboniferous of Ireland and Western Europe. Irish Naturalists' Journal 26 (11), 420-423.

XIA, F.S. \& LiU, X.L. 1986. Late Carboniferous bryozoans from the vicinity of Urumqi, Xinjiang. Memoirs of the Nanjing Institute of Geology and Palaeontology, Academia Sinica 22, 145-180. [in Chinese]

ZÁGORŠEK, K. 1993. New Carboniferous Bryozoa from Nagyvisnyó (Bükk Mts., Hungary). Földtani Közlöny 123(4), 417-440.

Zittel, K.A. von 1880. Handbuch der Palaeontologie. 1. Band. Palaeozoologie.1. Abtheilung. Protozoa, Coelenterata, Echinodermata und Molluscoidea. 765 pp. Oldenburg, München \& Leipzig. 